\title{
Numerical Methods for Optimal Harvesting Strategies in Random Environments under Partial Observations
}

\author{
Ky Tran, ${ }^{a}$ G. Yin ${ }^{b}$ \\ ${ }^{a}$ Department of Mathematics, Wayne State University, Detroit, Michigan 48202, kytran@wayne.edu. \\ ${ }^{\mathrm{b}}$ Department of Mathematics, Wayne State University, Detroit, Michigan 48202, gyin@math.wayne.edu.
}

\begin{abstract}
This work is concerned with optimal harvesting problems in random environments. In contrast to the existing literature, the Markov chain is hidden and can only be observed in a Gaussian white noise in our work. We first use the Wonham filter to estimate the Markov chain from the observable evolution of the given process so as to convert the original problem to a completely observable one. Then we treat the resulting optimal control problem. Because the problem is virtually impossible to solve in closed form, our main effort is devoted to developing numerical approximation algorithms. To approximate the value function and optimal strategies, Markov chain approximation methods are used to construct a discrete-time controlled Markov chain. Convergence of the algorithm is proved by weak convergence method and suitable scaling. A numerical example is provided to demonstrate the results.
\end{abstract}

Key words: Harvesting problem; Controlled regime-switching diffusion; Singular control; Partial observation; Wonham filter.

\section{Introduction}

This work focuses on optimal harvesting problems for ecosystems formulated by stochastic differential equations with regime switching represented by a continuoustime Markov chain. The problem belongs to the class of singular stochastic control problems motivated by the establishment of ecologically, environmentally, and economically reasonable wildlife management and harvesting policies. Recently, there has been a resurgent interest in determining the optimal harvesting strategies in the presence of stochastic fluctuations. Radner and Shepp (1996) derived the optimal strategy of a model for corporate strategy. Alvarez and Shepp (1998) studied the optimal harvesting plan for the stochastic VerhulstPearl logistic model. All the aforementioned works dealt with species living in an environment with a fixed configuration. Recently, Song, Stockbridge, \& Zhu (2011) and Tran and Yin (2015) considered singular control problems in random environments modeled by a Markov chain, in which Song, Stockbridge, \& Zhu (2011) dealt with a single species and Tran and Yin (2015) treated multiple species with interactions.

Suppose that there is a single species $X(t)$ whose growth is subject to the usual fluctuations as well as the abrupt changes of a random environment. Harvesting strategies are introduced to derive financial benefit as well as to control the growth of the population. Let $Z(t)$ denote the total amount harvested from the species up to time $t$. The goal is to find a harvesting strategy $Z(t)$ that maximizes the expected total discounted income from harvesting, up to the time when the population falls to a given threshold (e.g., extinction), which has the following economic interpretation. Let $X(t)$ be the value at time $t$ of asset/security/investment and $Z(t)$ represent the total amount paid in dividends up to time $t$. Then $\mathbb{R}_{+}=(0, \infty)$ can be regarded as the solvency set, and (13) becomes the problem of finding the optimal stream of dividends from the collection of assets until the time of bankruptcy; see Asmussen and Taksar (1997), Sotomayor and Cadenillas (2011), and Choulli, Taksar, \& Zhou (2003).

Harvesting may occur instantaneously, so it results in a singular stochastic control problem in the sense that the optimal harvesting strategy $Z(t)$ may not be absolutely continuous with respect to the Lebesgue measure of the time variable. For instance, if the discounted value and noise intensity are sufficiently large, driving the population to extinction instantly or chattering harvesting strategies might be optimal or near-optimal; see Alvarez and Shepp (1998), and Tran and Yin (2015). In contrast to regular stochastic control problems, in which the displacement of the state due to control is differentiable in time, the harvesting problem considered in this work allows the displacement to be discontinuous. To find the 
value function and the harvesting strategy, one usually solves a so-called Hamilton-Jacobi-Bellman (HJB) equation. However, for singular control problems with regime switching, the HJB equation is in fact a coupled system of nonlinear quasi-variational inequalities. A closed-form solution is virtually impossible to obtain. The Markov chain approximation methodology developed by Kushner and Dupuis (1992) becomes a viable alternative. As pointed out in Kushner and Dupuis (1992), probabilistic approach using the Markov chain approximation method for controlled diffusions has the following advantages. First, the Markov chain approximation method allows one to use physical insights derived from the dynamics of the controlled diffusion in obtaining a suitable approximation scheme. Second, the Markov chain approximation method does not require much regularity of the controlled processes (solutions of the controlled stochastic differential equations) nor does it rely on the properties of the associated HJB equations. Though it is well recognized the need of developing numerical approximation methods for singular control problems, the results are still scare. For singular controlled diffusions without regime switching, Budhiraja and Ross (2007), and Kushner and Martins (1991) are two of the representative works that carry out a convergence analysis using weak convergence and relaxed control formulation for singular control problems in the setting of Itô diffusions. Recently, some works have been devoted to numerical methods for singular controls with regime switching. Jin et al. (2012) developed numerical algorithms for finding optimal dividend pay-out and reinsurance policies under a generalized singular control formulation. A numerical algorithm for optimal dividend payment and investment strategies of regime-switching jump diffusion models with capital injections was then introduced in Jin, Yang, \& Yin (2013).

In our work, we focus on the harvesting problem for a partially observed system with a hidden Markov chain. So far, the work on numerical solutions has mostly concentrated on the case the Markov chain is observable. In reality, the environment (Markov chain) can often be only observed with noise. That is, at any given instance, the exact state of residency of the Markov chain is not known. Thus, we cannot see $\alpha(t)$ directly but only have noise-corrupted observation in the form of $\alpha(t)$ plus noise. An effective way to handle control problems of such partially observed systems is to converted them to completely observed ones, which can be done by using a Wonham filter (see, for example, Wonham (1965)). In the literature, the Wonham filters have been used widely to investigate control problems with partial observations; see Yang, Yin, \& Zhang (2014), Tran and Yin (2014) for applications in engineering, finance, and ecology. Note that our work is different from some problems in math finance, in which the $X(t)$ is taken to be the observation. Here we have another observation process in the form of the Markov chain observed in white noise. Such formulations appear in many networked con- trol problems, ecological systems, and cyber-physical systems. Compared to the aforementioned works on numerical methods for singular control problems, in the current work, we take a step towards more useful and realistic model where the Markov chain is unobservable. Although main ideas developed are crucial to the analysis of the current paper, there are key differences in the model that make our analysis more delicate. Using a Wonham filter, we convert the partially observed system into a fully observed controlled diffusion. We then design approximation procedures for the optimal strategies and the value function. We need to use a couple of step sizes $h=\left(h_{1}, h_{2}\right)$. The parameter $h_{1}>0$ is a discretization parameter for state variables, and $h_{2}>0$ is the step size for time variable. In the actual computing, the computations are involved due to the presence of the Wonham filter.

In contrast to the existing results, our new contributions in this paper are as follows. (i) We use Wonham's filter to formulate the harvesting problem in random environments when the Markov chain is only observable in white Gaussian noise. (ii) We convert the partially observed system to a fully observed system by replacing the unknown Markovian states by their posterior probability estimates. (iii) We develop numerical approximation schemes based on the Markov chain approximation method. Although Markov chain approximation techniques have been used extensively in various control problems, the work on combination of such method for a singular control problem with partial observation seems to be scarce to the best of our knowledge.

The rest of the paper is organized as follows. Section 2 begins with the problem formulation. Section 3 presents the numerical algorithm based on the Markov chain approximation method. Section 4 establishes the convergence of the algorithm. Finally, the paper is concluded with a numerical example for illustration together with further remarks in the last section.

\section{Formulation}

For $i=1, \ldots, r$, let $X^{i}(t)$ be the population size of the $i$ th species in the ecosystem at time $t$ and denote $X(t)=\left(X^{1}(t), \ldots, X^{r}(t)\right)^{\prime} \in \mathbb{R}^{r}$ (with $z^{\prime}$ denoting the transpose of $z \in \mathbb{R}^{r_{1} \times r_{2}}$ with $\left.r_{1}, r_{2} \geq 1\right)$. Suppose that species $X^{i}(t)$ live in random environments. In addition to the random fluctuations of the population, we also assume that the growth of the species is subject to abrupt changes within a finite number of configurations of the environment. For simplicity, we assume that the switching among different environments is memoryless and that the waiting time for the next switch is exponentially distributed. In fact, this phenomenon is frequently observed in nature; see Slatkin (1978), Yin and Zhu (2010). Thus we can model the random environments and other random factors in the ecological system by a continuous-time Markov chain $\alpha(t)$ taking values in $\mathcal{M}=\{1,2, \ldots, m\}$ with the generator given by $Q=\left(q^{i j}\right) \in \mathbb{R}^{m \times m}$. Assume throughout the paper 
that both the Markov chain $\alpha(t)$ and the $r$-dimensional standard Wiener process $w(\cdot)=\left(w^{1}(\cdot), \ldots, w^{r}(\cdot)\right)^{\prime}$ are defined on a complete filtered probability space $(\Omega, \mathcal{F}, \mathcal{F}(t), P)$, where $\{\mathcal{F}(t)\}$ is a filtration satisfying the usual condition (i.e., right continuous, increasing, and $\mathcal{F}(0)$ containing all the null sets).

In an effort to capture the salient feature that continuous dynamics and discrete events coexist in the ecosystem, we model the evolution in the absence of harvesting by the stochastic differential equation

$$
\begin{aligned}
& d X(t)=b(X(t), \alpha(t)) d t+\sigma(X(t), \alpha(t)) d w(t), \\
& X(0)=x_{0} \in \mathbb{R}_{+}^{r}, \alpha(0)=\alpha_{0} \in \mathcal{M},
\end{aligned}
$$

where $b(\cdot): \mathbb{R}^{r} \times \mathcal{M} \mapsto \mathbb{R}^{r}, \sigma(\cdot): \mathbb{R}^{r} \times \mathcal{M} \mapsto \mathbb{R}^{r \times r}$ are suitable functions. Furthermore, we assume that the Brownian motion $w(\cdot)$ and the Markov chain $\alpha(\cdot)$ are independent, a commonly used assumption in the literature. We attempt to answer the question: Can we solve optimal harvesting problems if the Markov chain is hidden and we can only treat a partially observed system? In particular, we cannot see $\alpha(t)$ directly but only have noise-corrupted observation in the form of $\alpha(t)$ plus noise. That is, we can observe the following process

$$
d y(t)=g(\alpha(t)) d t+\sigma_{0} d B(t), \quad y(0)=0,
$$

where $\sigma_{0}$ is a positive constant, $g: \mathcal{M} \mapsto \mathbb{R}$ is a one-toone function, $B(t)$ is a one-dimensional standard Brownian motion being independent of $w(t)$.

To proceed, we denote by $\mathbb{1}_{E}$ the indicator function of the event $E$, and use the following notation. For $j=$ $1, \ldots, m$

$$
\begin{aligned}
p^{j}(t) & :=\mathbb{1}_{\{\alpha(t)=j\}}, \\
\varphi^{j}(t) & :=P(\alpha(t)=j \mid y(s), 0 \leq s \leq t) .
\end{aligned}
$$

Since $\varphi^{j}(t)$ is the probability vector conditioned on the observation $\sigma\{y(s), 0 \leq s \leq t\}, \varphi^{j}(t) \geq 0$ and $\sum_{j=1}^{m} \varphi^{j}(t)=1$. Based on this property, it is sufficient to work with $\varphi(t):=\left(\varphi^{1}(t), \ldots, \varphi^{m-1}(t)\right)^{\prime}$. Such approach helps us to reduce one dimension in the actual computation. The actual state space for $\varphi(t)$ is

$$
S_{m-1}:=\left\{\varphi=\left(\varphi^{1}, \ldots, \varphi^{m-1}\right)^{\prime}: \varphi^{j} \geq 0, \sum_{j=1}^{m-1} \varphi^{j} \leq 1\right\} .
$$

For given functions $b(\cdot): \mathbb{R}^{r} \times \mathcal{M} \mapsto \mathbb{R}^{r}, \sigma(\cdot)$ : $\mathbb{R}^{r} \times \mathcal{M} \mapsto \mathbb{R}^{r \times r}$, and $g(\cdot): \mathcal{M} \rightarrow \mathbb{R}$, we define $\bar{b}(x, \varphi)=$ $\sum_{j=1}^{m} \varphi^{j} b(x, j), \bar{\sigma}(x, \varphi)=\sum_{j=1}^{m} \varphi^{j} \sigma(x, j), \bar{g}(\varphi)=$ $\sum_{j=1}^{m} \varphi^{j} g(j)$, for each $(x, \varphi) \in \mathbb{R}^{r} \times S_{m-1}$, where $S_{m-1}$ was defined in (4) and $\varphi^{m}=1-\sum_{i=1}^{m-1} \varphi^{j}$.

We first recall some results of Wonham's filter. As was mentioned, the Markov chain $\alpha(t)$ is observed through (2). It was proved in Wonham (1965) that the posterior probability $\varphi^{j}(\cdot)$ satisfies the following system of stochastic differential equations

$$
\left\{\begin{array}{l}
d \varphi^{j}(t)=\sigma_{0}^{-2}(g(j)-\bar{g}(\varphi(t))) \varphi^{j}(t) d y(t) \\
+\left[\sum_{k=1}^{m} q^{k i} \varphi^{k}(t)-\sigma_{0}^{-2}(g(j)-\bar{g}(\varphi(t))) \bar{g}(\varphi(t)) \varphi^{j}(t)\right] d t \\
\varphi^{j}(0)=\varphi_{0}^{j}, \quad j=1, \ldots, m
\end{array}\right.
$$

where $\left(\varphi_{0}^{1}, \ldots, \varphi_{0}^{m}\right)^{\prime}$ is the initial distribution of $\alpha(t)$. Introduce a one dimensional innovation process

$$
d \bar{w}(t)=\sigma_{0}^{-1}(d y(t)-\bar{g}(\varphi(t)) d t), \quad \bar{w}(0)=0 .
$$

Then the first $m-1$ equations in (5) can be rewritten as $d \varphi^{j}(t)=\sum_{k=1}^{m} q^{k j} \varphi^{k}(t) d t+\sigma_{0}^{-1} \varphi^{j}(t)(g(j)-\bar{g}(\varphi(t))) d \bar{w}(t)$.

With the use of $(3),(1)$ can be written as

$$
\begin{aligned}
X(t)= & x_{0}+\int_{0}^{t} \sum_{j=1}^{m} p^{j}(s) b(X(s), j) d s \\
& +\int_{0}^{t} \sum_{j=1}^{m} p^{j}(s) \sigma(X(s), j) d w(s) .
\end{aligned}
$$

Replacing the hidden state $p^{j}(t)$ by its estimate $\varphi^{j}(t)$ in (7), we obtain an estimated process of the original population process. For notational simplicity, we still use $X(t)$ to denote that estimated process. That is,

$X(t)=x_{0}+\int_{0}^{t} \bar{b}(X(s), \varphi(t)) d s+\int_{0}^{t} \bar{\sigma}(X(s), \varphi(t)) d w(s)$.

Let $Z(t)=\left(Z^{1}(t), \ldots, Z^{r}(t)\right)^{\prime} \in \mathbb{R}^{r}$, where $Z^{i}(t)$ denote the total number harvested (to be defined shortly) from the species $i$ up to time $t$. Then $\xi(t)=\left(\xi^{1}(t), \ldots, \xi^{r}(t)\right)^{\prime} \in$ $\mathbb{R}^{r}$, the population size of the harvested population, satisfies

$$
\begin{aligned}
\xi(t)=x_{0} & +\int_{0}^{t} \bar{b}(\xi(s), \varphi(t)) d s \\
& +\int_{0}^{t} \bar{\sigma}(\xi(s), \varphi(t)) d w(s)-Z(t),
\end{aligned}
$$

with initial data

$$
\xi(0-)=x_{0} \in \mathbb{R}_{+}^{r}, \quad \varphi(0)=\varphi_{0} \in S_{m-1} .
$$

At each time $t, \xi(t-)$ is the state before harvest starting at time $t$, while $\xi(t)$ is the state immediately after. Hence $\xi(0)$ may not be equal to $\xi(0-)$ due to an instantaneous harvest $Z(0)$ at time 0 . Throughout the paper we use the convention that $Z(0-)=0$. If $Z$ consists of an immediate harvest at time $t$, then this jump size is denoted by $\Delta Z(t):=Z(t)-Z(t-)$, and $Z^{c}(t):=Z(t)-\sum_{0 \leq s \leq t} \Delta Z(s)$ denotes the continuous part of $Z$. Since $Z(\cdot)$ is not required to be absolutely continuous with respect to the Lebesgue measure of the time variable, it is referred to as singular con- 
trol. Also note that $\Delta \xi(t):=\xi(t)-\xi(t-)=-\Delta Z(t)$ for any $t \geq 0$. Hence (6) and (9) form a controlled process $(\xi(t), \varphi(t)) \in \mathbb{R}^{r} \times S_{m-1}$ with complete observation and the initial condition $\left(x_{0}, \varphi_{0}\right)$.

An admissible harvesting strategy is a stochastic process $Z(t)$ satisfying the following conditions: (a) $Z(t)$ is right continuous, nonnegative, and nondecreasing with respect to $t$, (b) $Z(t)$ is adapted to $\sigma\{w(s), \varphi(s): 0 \leq s \leq$ $t\}$, augmented by the $P$-null sets, (c) $J\left(x_{0}, \varphi_{0}, Z\right)<\infty$, for any $\left(x_{0}, \varphi_{0}\right) \in \mathbb{R}_{+}^{r} \times S_{m-1}$, where $J(\cdot)$ is the functional defined below. (d) $\xi(t) \geq 0$, for any $t \leq \tau$, where $\tau=\inf \left\{s \geq 0: \xi_{i}(s)=0\right.$, for all $\left.i=1, \ldots, r\right\}$ is the extinction time of the system.

Note that $\tau$ might be infinite. If $\tau=\infty$ a.s., then the corresponding harvesting strategy belongs to the class of singular control with state constraints Song and Zhu (2012). Let $\mathcal{A}_{x_{0}, \varphi_{0}}$ denote the collection of all admissible harvesting strategies with initial conditions given by (10). Let $f^{i}(\cdot): \mathcal{M} \mapsto \mathbb{R}_{+}:=(0, \infty)$ represent the instantaneous marginal yields accrued from exerting the harvesting strategy $Z^{i}$ for species $i$, also known as the price of species $i$. Define $\bar{f}^{i}(\varphi)=\sum_{j=1}^{m} \varphi^{j} f^{i}(j)$ for each $\varphi \in S_{m-1}$, with $\varphi^{m}=1-\sum_{j=1}^{m-1} \varphi^{j}$. Then $\mathbb{R}_{+}$is regarded as the survival set of each species and we impose $\xi^{i}(t)=0$ for $t \geq \inf \left\{s \geq 0: \xi^{i}(s)=0\right\}$. For a fixed harvesting process $Z \in \overline{\mathcal{A}}_{x_{0}, \varphi_{0}}$, the expected total discounted value from harvesting is defined by

$$
J\left(x_{0}, \varphi_{0}, Z\right):=\sum_{i=1}^{r} E_{x_{0}, \varphi_{0}} \int_{0}^{\tau} e^{-a s} \bar{f}^{i}(\varphi(s)) d Z^{i}(s),
$$

where $a>0$ is the discounting factor and $E_{x_{0}, \varphi_{0}}$ denotes the expectation with respect to the probability law when the process $(\xi(t), \varphi(t))$ starts with initial condition $\left(x_{0}, \varphi_{0}\right)$. The goal is to maximize the expected total discounted value from harvesting and find an optimal harvesting strategy $Z^{*}$ such that

$$
J\left(x_{0}, \varphi_{0}, Z^{*}\right)=V\left(x_{0}, \varphi_{0}\right):=\sup _{Z \in \mathcal{A}_{x_{0}, \varphi_{0}}} J\left(x_{0}, \varphi_{0}, Z\right) .
$$

To proceed, we introduce the generator of the combined process $(x(t), \varphi(t))$. For any twice continuously differentiable function $W(\cdot, \cdot): \mathbb{R}^{r} \times S_{m-1} \mapsto \mathbb{R}$, we define

$$
\begin{aligned}
& \mathcal{L} W(x, \varphi)=\sum_{i=1}^{r} W_{x_{i}} \bar{b}^{i}(x, \varphi) \\
& +\frac{1}{2} \sum_{i, j=1}^{r} \bar{a}^{i j}(x, \varphi) W_{x^{i} x^{j}}(x, \varphi)+\sum_{j=1}^{m-1} W_{\varphi^{j}}(x, \varphi) \sum_{k=1}^{m} q^{k j} \varphi^{k} \\
& +\frac{1}{2 \sigma_{0}^{2}} \sum_{j=1}^{m-1}\left[\sum_{k=1}^{m-1} \varphi^{j} \varphi^{k}(g(j)-\bar{g}(\varphi))(g(k)-\bar{g}(\varphi))\right] W_{\varphi^{j} \varphi^{k}},
\end{aligned}
$$

where partial derivatives are evaluated at $(x, \varphi)$, and

$$
\begin{aligned}
& \bar{b}(x, \varphi)=\left(\bar{b}^{1}(x, \varphi), \ldots, \bar{b}^{r}(x, \varphi)\right)^{\prime} \in \mathbb{R}^{r}, \\
& \bar{a}(x, \varphi)=\left(\bar{a}^{i j}(x, \varphi)\right)=\bar{\sigma}(x, \varphi) \bar{\sigma}^{\prime}(x, \varphi) \in \mathbb{R}^{r \times r} .
\end{aligned}
$$

Our standing assumptions are as follows.

(A1) $\bar{b}(\cdot, \cdot)$ and $\bar{\sigma}(\cdot, \cdot)$ satisfy the usual local Lipschitz condition and there exists a positive constant $C_{1}$ such that

$x^{\prime} \bar{b}(x, \varphi)+|\bar{\sigma}(x, \varphi)|^{2} \leq C_{1}\left(|x|^{2}+1\right),(x, \varphi) \in \mathbb{R}^{r} \times S_{m-1}$.

(A2) There exists a positive constant $C_{2}$ such that $\bar{a}(x, \varphi)-C_{2} I_{r}$ is positive definite for each $(x, \varphi) \in$ $\mathbb{R}^{r} \times S_{m-1}$, and for $i=1, \ldots, r$,

$$
\bar{a}^{i i}(x, \varphi)-\sum_{j: j \neq i}\left|\bar{a}^{i j}(x, \varphi)\right| \geq 0,(x, \varphi) \in \mathbb{R}^{r} \times S_{m-1},
$$

where $I_{r}$ is the $r \times r$ identity matrix.

Under (A1), for any initial condition $\left(x_{0}, \varphi_{0}\right) \in \mathbb{R}_{+}^{n} \times$ $S_{m-1}$, the system (6)-(8) has the unique global solution $(x(t), \varphi(t)) \in \mathbb{R}^{r} \times S_{m-1}$ for all $t \geq 0$; see Yin and Zhu (2010). Assumption (A2) is imposed for convenience. There are several ways of relaxing the condition (15) for which we refer to Kushner and Martins (1991). Meanwhile, the first condition in (A2) is a non-degeneracy requirement for the diffusion part. If it does not hold, one can use a trick in Kushner and Martins (1991)(p.288289) which requires more complex notation and the use of another Brownian motion.

Before proceeding further, recall that if the value functions are sufficiently smooth, they are solutions of the following system of HJB equations (see Song et al. (2011))

$$
\begin{aligned}
& \max \left((\mathcal{L}-r) W(x, \varphi), \bar{f}^{i}(\varphi)-W_{x^{i}}(x, \varphi), i=1, \ldots, r\right) \\
& \quad=0, \quad(x, \varphi) \in \mathbb{R}_{+}^{r} \times S_{m-1}, \\
& W(x, \varphi)=0, \quad(x, \varphi)=\{0\} \times S_{m-1} .
\end{aligned}
$$

Using the same argument as in Song et al. (2011), one can also establish a verification theorem that leads to an optimal harvesting strategy. However, due to the presence of partial observation, it is very difficult to find value functions and optimal harvesting policies explicitly except for some special cases. Our task to follow is to construct a numerical procedure for solving the optimal control problem.

\section{Numerical Algorithm}

The basic idea behind the numerical method is to find a controlled Markov chain in discrete time to approximate the controlled diffusions. The method is similar to Budhiraja and Ross (2007), Jin et al. (2012), Kushner and Martins (1991), Kushner and Dupuis (1992). However, some important modifications are required due to the presence of a combination of singular control and partial observation. 


\subsection{Approximation Algorithm of the Wonham Filter}

In this section, we deal with the numerical algorithms for the two components system. We begin with the Wonham filter equations. To construct approximation algorithms, one may wish to discretize the stochastic differential equations (5) directly. However, such a procedure is numerically unstable due to the white noise perturbations. To overcome this difficulty, we use the approach suggested in Yin and Zhang (2006) (Section 8.4), in which a logarithm transformation is used to transform the stochastic differential equations and then a numerical procedure for the transformed system is constructed. Let $v^{j}(t):=\ln \varphi^{j}(t)$ for $t \geq 0$ and $j=1, \ldots, m$. It follows that $\varphi^{j}(t)=e^{v^{j}(t)}$. An application of Itô's formula to (6) leads to that for each $j=1, \ldots, m$,

$$
\left\{\begin{aligned}
d v^{j}(t)= & {\left[\sum_{k=1}^{m} q^{k j} \frac{\varphi^{k}(t)}{\varphi^{j}(t)}-\frac{1}{2 \sigma_{0}^{2}}(g(j)-\bar{g}(\varphi(t)))^{2}\right] d t } \\
& +\frac{1}{2 \sigma_{0}}(g(j)-\bar{g}(\varphi(t))) d \bar{w}(t) \\
v^{j}(0)= & \ln \left(\varphi_{0}^{j}\right) .
\end{aligned}\right.
$$

We use the constant step size $h_{2}>0$ for time variable. There are a couple of ways to construct discretetime approximation of Wonham's filters. One possibility is the approach in Yin and Zhang (2006) (see Section 8.4). Here employing the approximation algorithm constructed in Yang et al. (2014), we can discretize (17) as follows.

$$
\left\{\begin{array}{l}
v_{0}^{h_{2}, j}=\ln \left(\varphi_{0}^{j}\right), \quad \varphi_{0}^{h_{2}, j}=\varphi_{0}^{j}, \\
\bar{g}_{n}^{h_{2}}=\sum_{k=1}^{m} g(k) \varphi_{n}^{h_{2}, k}, \\
r_{n}^{h_{2}, j}=\sum_{k=1}^{m} q^{k j} \frac{\varphi_{n}^{h_{2}, k}}{\varphi_{n}^{h_{2}, j}}-\frac{1}{2 \sigma_{0}^{2}}\left(g(j)-\bar{g}_{n}^{h_{2}}\right)^{2}, \\
v_{n+1}^{h_{2}, j}=v_{n}^{h_{2}, j}+h_{2} r_{n}^{h_{2}, j}+\frac{1}{\sigma_{0}}\left(g(j)-\bar{g}_{n}^{h_{2}}\right) \sqrt{h_{2}} \zeta_{n}, \\
\varphi_{n+1}^{h_{2}, j}=\exp \left(v_{n+1}^{h_{2}, j}\right) / \sum_{k=1}^{m} \exp \left(v_{n+1}^{h_{2}, k}\right),
\end{array}\right.
$$

where $\left\{\zeta_{n}\right\}$ is a sequence of independent and identically distributed random variables. Let $\left\{\varphi_{n}^{h_{2}}\right\}$ be the sequence of discretized posterior probabilities in which $\varphi_{n}^{h_{2}}=\left(\varphi_{n}^{h_{2}, 1}, \ldots, \varphi_{n}^{h_{2}, m-1}\right)^{\prime}$. We use the last equation in (18) to reinforce that $\varphi_{n}^{h_{2}} \in S_{m-1}$ for each $n$. Such property is convenient for convergence verifications of the problem under consideration. Note that $\varphi_{n}^{h_{2}, j}$ appeared as the denominator in (18) and we have focused on the case that $\varphi_{n}^{h_{2}, j}$ stays away from 0 . A modification can be made to take into consideration the case of $\varphi_{n}^{h_{2}, j}=0$; see Yin and Zhang (2006) for details. The conditions needed for the convergence analysis of the Wonham filter approximation are as follows.

(A3) $\left\{\zeta_{n}\right\}$ is a sequence of independent and identically distributed random variables and $\gamma>0$ is a constant such that $E \zeta_{n}=0, E \zeta_{n}^{2}=1, E\left|\zeta_{n}\right|^{2+\gamma}<\infty$.

\subsection{Approximation Algorithm of the Harvested Process}

In what follows, we construct a discrete-time finite state Markov chain to approximate the harvested process $\xi(t)$. Let $h_{1}>0$ be a discretization parameter for state variable, and recall that $h_{2}>0$ is the step size for time variable that we used above. Denote $h=\left(h_{1}, h_{2}\right)$. In the later presentation, for simplicity, we also use $\varphi^{h}$ for $\varphi^{h_{2}}$. By writing $h \rightarrow 0$, we mean $h=\left(h_{1}, h_{2}\right) \rightarrow(0,0)$. Let $U \in(0, \infty)$ be an upper bound introduced for numerical purpose only. Moreover, assume without loss of generality that the boundary point $U$ is an integer multiple of $h_{1}$. Define

$L_{h_{1}}:=\left\{\left(k^{1} h_{1}, \ldots, k^{r} h_{1}\right)^{\prime}: k^{i}=0, \pm 1, \ldots\right\} \cap\left[0, U+h_{1}\right]^{r}$.

Let $\left\{\xi_{n}^{h}: n=0,1, \ldots\right\}$ be a discrete-time controlled Markov chain with state space $L_{h_{1}}$ such that the controlled Markov chain well approximates the local behavior of the controlled diffusion (9). We define $\Delta \xi_{n}^{h}=$ $\xi_{n+1}^{h}-\xi_{n}^{h}$. At any discrete-time step $n$, we can either exercise a harvesting action, a reflection action, or not to apply a control. Each of these is described precisely in what follows. If we do not apply a control, then the increment $\Delta \xi_{n}^{h}$ is to "behave like" an increment of $\int \bar{b} d t+\int \bar{\sigma} d w$ over a small time interval. We call this a "diffusion step". We can write

$$
\begin{aligned}
\Delta \xi_{n}^{h}= & \Delta \xi_{n}^{h} I_{\{\text {diffusion step at } n\}}+\Delta \xi_{n}^{h} I_{\{\text {harvesting step at } n\}} \\
& +\Delta \xi_{n}^{h} I_{\{\text {reflection step at } n\}} .
\end{aligned}
$$

The chain and the control will be chosen so that there is exactly one term on the right-hand side of (19) is positive. Let $\pi^{h}=\left(\pi_{0}^{h}, \pi_{1}^{h}, \ldots\right)$ denote the sequence of control actions at time $0,1, \ldots$ We take $\pi_{n}^{h}=-i, 0$, or $i$, if we exercise a reflection on species $i$, not to apply a control, or harvesting action on species $i$ at time $n$, respectively. Let $\left\{\psi_{n}^{h}\right\}$ be the sequence of discretized posterior probabilities associated with $\left\{\xi_{n}^{h}\right\}$, to be defined shortly. Let $E_{x, \psi, n}^{h, \pi}, \operatorname{Cov}_{x, \psi, n}^{h, \pi}$ denote the conditional expectation and covariance given by $\left\{\xi_{k}^{h}, \psi_{k}^{h}, \pi_{k}^{h}, k \leq n, \xi_{n}^{h}=x, \psi_{n}^{h}=\right.$ $\left.\psi, \pi_{n}^{h}=\pi\right\}$, respectively. By stating that $\left\{\xi_{n}^{h}\right\}$ is a controlled discrete-time Markov chain on a finite state space $L_{h_{1}}$ with transition probabilities from state $x$ to another state $y$, denoted by $p^{h}((x, y) \mid \pi, \psi)$, we mean that the transition probabilities are functions of control action $\pi$ and posterior probability $\psi$. The sequence $\left\{\xi_{n}^{h}\right\}$ is said to be locally consistent with respect to (9) if it satisfies

$$
\begin{aligned}
& E_{x, \psi, n}^{h, 0} \Delta \xi_{n}^{h}=\bar{b}(x, \psi) h_{2}+o\left(h_{2}\right), \\
& \operatorname{Cov}_{x, \psi}^{h, 0} \Delta \xi_{n}^{h}=\bar{a}(x, \psi) h_{2}+o\left(h_{2}\right), \\
& \sup _{n, \omega}\left|\Delta \xi_{n}^{h}\right| \rightarrow 0, \quad h \rightarrow 0 .
\end{aligned}
$$

We define $\psi_{0}=\varphi_{0}$, where $\varphi_{0}$ is the initial value of the Wonham filter. For each $n \geq 0$, if $\pi_{n}^{h}=i$, we assume that the harvesting amount for species $i$ at time $n$ is 
$h_{1}$. Hence the harvesting amount for the chain at time $n$ is $\Delta z_{n}^{h}=\xi_{n}^{h}-\xi_{n+1}^{h}=h_{1} \sum_{i=1}^{r} \mathbf{e}_{i} \mathbb{1}_{\left\{\pi_{n}^{h}=i\right\}}$. If the $i$ th component of $\xi_{n}^{h}$ equals $U+h_{1}$, a reflection step is exerted definitely. i.e., $\pi_{n}^{h}=-i$. Moreover, we require that reflection takes the $i$ th component of the chain from $U+h_{1}$ to $U$. We denote by $\Delta g_{n}^{h}$ the random vector that is the reflection amount for the chain at time $n$, then $\Delta g_{n}^{h}=\xi_{n}^{h}-\xi_{n+1}^{h}=h_{1} \sum_{i=1}^{r} \mathbf{e}_{i} \mathbb{1}_{\left\{\pi_{n}^{h}=-i\right\}}$. Because the reflection and harvesting terms change the population process instantaneously, we define $\psi_{n+1}^{h}=\psi_{n}^{h}$ if $\pi_{n}^{h}=i$ or $\pi_{n}^{h}=-i$. If $\pi_{n}^{h}=0$ and $\psi_{n}^{h}=\varphi_{k}^{h}$ for some integer $k$, we define $\psi_{n+1}^{h}=\varphi_{k+1}^{h}$. Hence we have defined the sequences $\left\{\psi_{n}^{h}\right\}$ and $\left\{\xi_{n}^{h}\right\}$ recursively. As described above, the control at each step, is specified by the choice of an action: diffusion, harvesting, or reflection. Denote $\mathcal{F}_{n}^{h}=\sigma\left\{\xi_{k}^{h}, \psi_{k}^{h}, \pi_{k}^{h}, k \leq n\right\}$. The sequence $\pi^{h}$ is said to be admissible if $\pi_{n}^{h}$ is $\sigma\left\{\xi_{0}^{h}, \ldots, \xi_{n}^{h}, \psi_{0}^{h}, \ldots, \psi_{n}^{h}, \pi_{0}^{h}, \ldots, \pi_{n-1}^{h}\right\}-$ adapted, and for any $x \in L_{h_{1}}$, we have

$$
\begin{aligned}
& P\left\{\xi_{n+1}^{h}=x \mid \mathcal{F}_{n}^{h}\right\}=P\left\{\xi_{n+1}^{h}=x \mid \xi_{n}^{h}, \psi_{n}^{h}, \pi_{n}^{h}\right\} \\
& \quad=p^{h}\left(\xi_{n}^{h}, x \mid \psi_{n}^{h}, \pi_{n}^{h}\right), \\
& P\left\{\pi_{n}^{h}=-i \mid \xi_{n}^{h, i}=U+h_{1}, \mathcal{F}_{n}^{h}\right\}=1, \\
& P\left\{\pi_{n}^{h}=-i \mid \xi_{n}^{h, i} \leq U, \mathcal{F}_{n}^{h}\right\}=0,
\end{aligned}
$$

where $\xi_{n}^{h, i}$ denote the $i$ th component of the vector $\xi_{n}^{h}$. The class of all admissible control sequences for initial state $\left(x_{0}, \varphi_{0}\right)$ will be denoted by $\mathcal{A}_{x_{0}, \varphi_{0}}^{h}$. For each triple $(x, \psi, i) \in L_{h_{1}} \times S_{m-1} \times\{0, i,-i: i=1, \ldots, r\}$, we first define a family of the interpolation intervals $\Delta t^{h}(x, \psi, i)$. For diffusion steps, if the state of the chain is $x$ and the state of the discretized Wonham's filter is $\psi, \Delta t^{h}(x, \psi, i=0)$ will be taken to be $h_{2}$; whereas for harvesting steps and reflection steps, $\Delta t^{h}(x, \psi, i)$ will be taken to be 0 . This reflects the fact that for the controlled diffusion process, reflection and harvesting terms can change the state instantaneously. Therefore, we define

$$
\begin{aligned}
& \Delta t^{h}(x, \psi, i)=h_{2} I_{\{i=0\}}, t_{0}^{h}=0, \\
& \Delta t_{k}^{h}=\Delta t^{h}\left(\xi_{k}^{h}, \psi_{k}^{h}, \pi_{k}^{h}\right), t_{n}^{h}=\sum_{k=0}^{n-1} \Delta t_{k}^{h} .
\end{aligned}
$$

Let $\eta_{h}:=\inf \left\{n: \xi_{n}^{h} \notin L_{h_{1}}\right\}$. Then the first exit time of $\xi_{n}^{h}$ from $L_{h_{1}}$ is $\tau_{h}:=t_{\eta_{h}}^{h}$. For $(x, \varphi) \in L_{h_{1}} \times S_{m-1}$ and $\pi^{h}$ is admissible, the cost function for the controlled Markov chain is defined as

$$
J^{h}\left(x_{0}, \varphi_{0}, z^{h}\right)=\sum_{i=1}^{r} E \sum_{k=1}^{\eta^{h}-1} e^{-a t_{k}^{h}} \bar{f}^{i}\left(\psi_{k}^{h}\right) \Delta z_{k}^{h, i},
$$

where $\Delta z_{k}^{h}=\left(\Delta z_{k}^{h, 1}, \ldots, \Delta z_{k}^{h, r}\right)^{\prime}$. The value function of the controlled Markov chain is

$$
V^{h}\left(x_{0}, \varphi_{0}\right)=\sup _{z^{h}} J^{h}\left(x_{0}, \varphi_{0}, z^{h}\right) .
$$

\subsection{Transition Probabilities and Local Consistency}

Now we define the approximation to the first and the second derivatives of $V(\cdot, \cdot)$ by finite difference method using stepzise $h_{1}>0$ for the state variable as

$$
\begin{aligned}
& V(x, \varphi) \rightarrow V^{h}(x, \varphi), \\
& V_{x^{i}}(x, \varphi) \rightarrow \frac{V^{h}\left(x+h_{1} \mathbf{e}_{\mathbf{i}}, \varphi\right)-V^{h}(x, \varphi)}{h_{1}}, \text { if } \bar{b}^{i}(x, \varphi) \geq 0, \\
& V_{x^{i}}(x, \varphi) \rightarrow \frac{V^{h}(x, \varphi)-V^{h}\left(x-h_{1} \mathbf{e}_{\mathbf{i}}, \varphi\right)}{h_{1}}, \text { if } \bar{b}^{i}(x, \varphi)<0, \\
& V_{\left(x^{i}\right)^{2}}(x, \varphi) \rightarrow \frac{V^{h}\left(x+h_{1} \mathbf{e}_{\mathbf{i}}, \varphi\right)-2 V^{h}(x, \varphi)+V^{h}\left(x-h_{1} \mathbf{e}_{\mathbf{i}}, \varphi\right)}{h_{1}^{2}}, \\
& V_{x^{i} x^{j}}(x, \varphi) \rightarrow \frac{2 V^{h}(x, \varphi)+V^{h}\left(x+h_{1} \mathbf{e}_{\mathbf{i}}+h_{1} \mathbf{e}_{\mathbf{j}}, \varphi\right)}{2 h_{1}^{2}} \\
& +\frac{V^{h}\left(x-h_{1} \mathbf{e}_{\mathbf{i}}-h_{1} \mathbf{e}_{\mathbf{j}}, \varphi\right)-V^{h}\left(x+h_{1} \mathbf{e}_{\mathbf{i}}, \varphi\right)-V^{h}\left(x-h_{1} \mathbf{e}_{\mathbf{i}}, \varphi\right)}{2 h_{1}^{2}} \\
& -\frac{V^{h}\left(x+h_{1} \mathbf{e}_{\mathbf{j}}, \varphi\right)+V^{h}\left(x-h_{1} \mathbf{e}_{\mathbf{j}}, \varphi\right)}{2 h_{1}^{2}}, \quad \text { if } \bar{a}^{i j}(x, \varphi) \geq 0, \\
& V_{x^{i} x^{j}}(x, \varphi) \rightarrow-\frac{2 V^{h}(x, \varphi)+V^{h}\left(x+h_{1} \mathbf{e}_{\mathbf{i}}-h_{1} \mathbf{e}_{\mathbf{j}}, \varphi\right)}{2 h_{1}^{2}} \\
& +\frac{V^{h}\left(x+h_{1} \mathbf{e}_{\mathbf{i}}, \varphi\right)+V^{h}\left(x-h_{1} \mathbf{e}_{\mathbf{i}}, \varphi\right)-V^{h}\left(x-h_{1} \mathbf{e}_{\mathbf{i}}+h_{1} \mathbf{e}_{\mathbf{j}}, \varphi\right)}{2 h_{1}^{2}} \\
& +\frac{V^{h}\left(x+h_{1} \mathbf{e}_{\mathbf{j}}, \varphi\right)+V^{h}\left(x-h_{1} \mathbf{e}_{\mathbf{j}}, \varphi\right)}{2 h_{1}^{2}}, \quad \text { if } \bar{a}^{i j}(x, \varphi)<0,
\end{aligned}
$$

where $\mathbf{e}_{\mathbf{i}} \in \mathbb{R}^{r}$ denotes the unit vector in the $i$ th direction, $i=1, \ldots, r$. For the first and the second derivatives with respect to the posterior probability, we use similar approximations. We proceed to figure out the transition probabilities. We define for a real number $a$ that $a^{+}=\max \{a, 0\}, a^{-}=-\min \{0, a\}$. To find transition probabilities of the controlled Markov chain, we plug all the necessary expressions into the first part of system (16), then use the symmetry of the $\bar{a}(x, \varphi)$ matrix, combine like terms and divide by the coefficient of $V^{h}(x, \varphi)$. The transition probabilities are coefficients of the resulting equation. For $x \in L_{h_{1}}$ and $\psi \in S_{m-1}$, we define the transition probabilities at diffusion steps in the following way,

$$
\begin{aligned}
& p^{h}\left(x, x+h_{1} \mathbf{e}_{\mathbf{i}} \mid \psi, \pi=0\right) \\
& =\frac{\left(\bar{a}^{i i}(x, \psi) / 2-\sum_{j: j \neq i}\left|\bar{a}^{i j}(x, \psi)\right| / 2+\bar{b}^{i+}(x, \psi) h_{1}\right) h_{2}}{h_{1}^{2}},
\end{aligned}
$$

$$
\begin{aligned}
& p^{h}\left(x, x-h_{1} \mathbf{e}_{\mathbf{i}} \mid \psi, \pi=0\right) \\
& =\frac{\left(\bar{a}^{i i}(x, \psi) / 2-\sum_{j: j \neq i}\left|\bar{a}^{i j}(x, \psi)\right| / 2+\bar{b}^{i^{-}}(x, \psi) h_{1}\right) h_{2}}{h_{1}^{2}}, \\
& p^{h}\left(x, x+h_{1} \mathbf{e}_{\mathbf{i}}+h_{1} \mathbf{e}_{\mathbf{j}} \mid \psi, \pi=0\right) \\
& =p^{h}\left(x, x-h_{1} \mathbf{e}_{\mathbf{i}}-h_{1} \mathbf{e}_{\mathbf{j}} \mid \psi, \pi=0\right)=\frac{\bar{a}^{i j+}(x, \psi) h_{2}}{2 h_{1}^{2}},
\end{aligned}
$$




$$
\begin{aligned}
& p^{h}\left(x, x+h_{1} \mathbf{e}_{\mathbf{i}}-h_{1} \mathbf{e}_{\mathbf{j}} \mid \psi, \pi=0\right) \\
& =p^{h}\left(x, x-h_{1} \mathbf{e}_{\mathbf{i}}+h_{1} \mathbf{e}_{\mathbf{j}} \mid \psi, \pi=0\right)=\frac{\bar{a}^{i j^{-}}(x, \psi) h_{2}}{2 h_{1}^{2}}, \\
& p^{h}(x, x \mid \psi, \pi=0)=1-\sum p^{h}\left(x, x \pm h_{1} \mathbf{e}_{\mathbf{i}} \mid \psi, \pi=0\right) \\
& \quad-\sum p^{h}\left(x, x+h_{1} \mathbf{e}_{\mathbf{i}} \pm h_{1} \mathbf{e}_{\mathbf{j}} \mid \psi, \pi=0\right) .
\end{aligned}
$$

Assumption (A2) guarantees that $p^{h}\left(x, x+h_{1} \mathbf{e}_{\mathbf{i}} \mid \psi, \pi=\right.$ $0) \geq 0$ and $p^{h}\left(x, x-h_{1} \mathbf{e}_{\mathbf{i}} \mid \psi, \pi=0\right) \geq 0$ for all $(x, \psi) \in$ $L_{h_{1}} \times S_{m-1}$. Moreover, by choosing proper $h_{1}$ and $h_{2}$ (for instance, $h_{2}=o\left(h_{1}^{2}\right)$ ), we can reasonably assume that $p^{h}(x, x \mid \psi, \pi=0) \geq 0$, that is, the transition probabilities in (24)-(27) are well-defined. At reflection steps and harvesting steps, we define

$$
p^{h}\left(x, x-h_{1} \mathbf{e}_{\mathbf{i}} \mid \psi, \pi= \pm i\right)=1 .
$$

The definition of the transition function at 0 is not important since in the analysis of the control problem, the chain will be stopped the first time it hits 0 . In (24)-(27), we implicitly set that $\bar{a}^{i j}(x, \psi)=\bar{a}^{j i}(x, \psi)=\bar{b}^{i}(x, \psi)=$ 0 for all $j=1, \ldots, r$ if $x^{i}=0$ and $p^{h}(0,0 \mid \psi, \pi=0)=1$. Let $\xi_{n}^{h, i}$ denote the $i$ th component of the vector $\xi_{n}^{h}$. Using the above transition probabilities we can verify that $E_{x, \psi, n}^{h, 0} \Delta \xi_{n}^{h, i}=\bar{b}^{i}(x, \psi) h_{2}$. Moreover,

$$
E_{x, \psi, n}^{h, 0}\left(\Delta \xi_{n}^{h, i} \Delta \xi_{n}^{h, j}\right)=\bar{a}^{i j}(x, \psi) h_{2}+o\left(h_{2}\right), \quad h_{2} \rightarrow 0 .
$$

The local consistence of the controlled Markov chain $\left\{\xi_{n}^{h}\right\}$ follows.

\section{Convergence}

\subsection{Interpolation and Time Rescaling}

One of the main goals of the study is to show that the value function of the controlled Markov chain defined in (23) converges, as $h=\left(h_{1}, h_{2}\right) \rightarrow(0,0)$, to the value function of the limit control problem. This convergence result allows for the computation of near optimal policies for the control problem by numerical method. We next introduce the continuous-time interpolation and time rescaling techniques that will be used in the proof of our main convergence result. The continuous-time interpolations of various processes will be constructed to be piecewise constant on the time interval $\left[t_{n}^{h}, t_{n+1}^{h}\right), n \geq 0$. For use in this construction, we define $n^{h}(t)=\max \{n$ : $\left.t_{n}^{h} \leq t\right\}, t \geq 0$. We first define discrete time processes associated with the controlled Markov chain as follows. Let $z_{0}^{h}=g_{0}^{h}=B_{0}^{h}=M_{0}^{h}=0$ and define for $n \geq 1$,

$$
\begin{aligned}
& z_{n}^{h}=\sum_{k=0}^{n-1} h_{1} \mathbb{1}_{\left\{\pi_{k}^{h} \geq 1\right\}}, M_{n}^{h}=\sum_{k=0}^{n-1}\left(\Delta \xi_{k}^{h}-E_{k}^{h} \Delta \xi_{k}^{h}\right) \mathbb{1}_{\left\{\pi_{k}^{h}=0\right\}}, \\
& g_{n}^{h}=\sum_{k=0}^{n-1} h_{1} \mathbb{1}_{\left\{\pi_{k}^{h} \leq-1\right\}}, B_{n}^{h}=\sum_{k=0}^{n-1} \mathbb{1}_{\left\{\pi_{k}^{h}=0\right\}} E_{k}^{h} \Delta \xi_{k}^{h} .
\end{aligned}
$$

The piecewise constant interpolations, denoted by $\left(\xi^{h}(\cdot), \psi^{h}(\cdot), z^{h}(\cdot), g^{h}(\cdot), B^{h}(\cdot), M^{h}(\cdot)\right)$ are naturally defined as

$$
\begin{aligned}
& \xi^{h}(t)=\xi_{n^{h}(t)}^{h}, \quad \psi^{h}(t)=\psi_{n^{h}(t)}^{h}, \\
& z^{h}(t)=z_{n^{h}(t)}^{h}, \quad g^{h}(t)=g_{n^{h}(t)}^{h}, \\
& B^{h}(t)=B_{n^{h}(t)}^{h}, \quad M^{h}(t)=M_{n^{h}(t)}^{h} \quad t \geq 0 .
\end{aligned}
$$

Define $\mathcal{F}^{h}(t)=\sigma\left\{\xi^{h}(s), \psi^{h}(s), g^{h}(s), z^{h}(s): s \leq t\right\}=$ $\mathcal{F}_{n^{h}(t)}^{h}$. Using the representation of diffusion steps, harvesting steps, reflection steps in (19), we obtain

$$
\begin{aligned}
\xi_{n}^{h}=x_{0} & +\sum_{k=0}^{n-1} \Delta \xi_{k}^{h} \mathbb{1}_{\left\{\pi_{k}^{h} \leq-1\right\}}+\sum_{k=0}^{n-1} \Delta \xi_{k}^{h} \mathbb{1}_{\left\{\pi_{k}^{h} \geq 1\right\}} \\
& +\sum_{k=0}^{n-1} \Delta \xi_{k}^{h} \mathbb{1}_{\left\{\pi_{k}^{h}=0\right\}}
\end{aligned}
$$

Using the interpolations defined above, we have

$$
\xi^{h}(t)=x_{0}+B^{h}(t)+M^{h}(t)-z^{h}(t)-g^{h}(t) .
$$

Recall that $\Delta t_{k}^{h}=h_{2}$ if $\pi_{k}^{h}=0$ and $\Delta t_{k}^{h}=0$ if $\pi_{k}^{h} \geq 1$ or $\pi_{k}^{h} \leq-1$. It follows that

$$
\begin{aligned}
B^{h}(t) & =\sum_{k=0}^{n^{h}(t)-1} \bar{b}\left(\xi_{k}^{h}, \psi_{k}^{h}\right) \Delta t_{k}^{h} \\
& =\int_{0}^{t} \bar{b}\left(\xi^{h}(s), \psi^{h}(s)\right) d s-\int_{t_{n^{h}(t)}^{t}} \bar{b}\left(\xi^{h}(s), \psi^{h}(s)\right) d s \\
& =\int_{0}^{t} \bar{b}\left(\xi^{h}(s), \psi^{h}(s)\right) d s+\varepsilon_{1}^{h}(t),
\end{aligned}
$$

with $\left\{\varepsilon_{1}^{h}(\cdot)\right\}$ is an $\mathcal{F}^{h}(t)$-adapted process satisfying

$$
\lim _{h \rightarrow 0} \sup _{t \in\left[0, T_{0}\right]} E\left|\varepsilon_{1}^{h}(t)\right|=0, \quad \text { for any } 0<T_{0}<\infty .
$$

We now attempt to represent $M^{h}(\cdot)$ in a form similar to the diffusion term in (9). Factor

$$
\bar{a}(x, \varphi)=\bar{\sigma}(x, \varphi) \bar{\sigma}^{\prime}(x, \varphi)=P(x, \varphi) D^{2}(x, \varphi) P^{\prime}(x, \varphi),
$$

where $D(\cdot)=\operatorname{diag}\left\{d^{1}(\cdot), \ldots, d^{r}(\cdot)\right\}, P(\cdot)$ is an orthogonal matrix. By assumption (A2), $\inf _{(x, \varphi)} d^{i}(x, \varphi)>0$ for all $i=1, \ldots, r$. Define $D_{0}(\cdot)=\operatorname{diag}\left\{1 / d^{1}(\cdot), \ldots, 1 / d^{r}(\cdot)\right\}$. Define $w^{h}(\cdot)$ by

$$
\begin{aligned}
& w^{h}(t)=\int_{0}^{t} D_{0}\left(\xi^{h}(s), \psi^{h}(s)\right) P^{\prime}\left(\xi^{h}(s), \psi^{h}(s)\right) d M^{h}(s) \\
& =\sum_{k=0}^{n^{h}(t)-1} D_{0}\left(\xi_{k}^{h}, \psi_{k}^{h}\right) P^{\prime}\left(\xi_{k}^{h}, \psi_{k}^{h}\right)\left(\Delta \xi_{k}^{h}-E_{k}^{h} \Delta \xi_{k}^{h}\right) \mathbb{1}_{\left\{\pi_{k}^{h}=0\right\}} .
\end{aligned}
$$

Then we can write

$$
M^{h}(t)=\int_{0}^{t} \bar{\sigma}\left(\xi^{h}(s), \psi^{h}(s)\right) d w^{h}(s)+\varepsilon_{2}^{h}(t),
$$

with $\left\{\varepsilon_{2}^{h}(\cdot)\right\}$ is an $\mathcal{F}^{h}(t)$-adapted process satisfying

$$
\lim _{h \rightarrow 0} \sup _{t \in\left[0, T_{0}\right]} E\left|\varepsilon_{2}^{h}(t)\right|=0, \quad \text { for any } 0<T_{0}<\infty .
$$


Using (33) and (35), we can write (32) as

$$
\begin{aligned}
& \xi^{h}(t)=x_{0}+\int_{0}^{t} \bar{b}\left(\xi^{h}(s), \psi^{h}(s)\right) d s \\
& +\int_{0}^{t} \bar{\sigma}\left(\xi^{h}(s), \psi^{h}(s)\right) d w^{h}(s)-z^{h}(t)-g^{h}(t)+\varepsilon^{h}(t),
\end{aligned}
$$

with $\varepsilon^{h}(\cdot)$ is an $\mathcal{F}^{h}(t)$-adapted process satisfying

$$
\lim _{h \rightarrow 0} \sup _{t \in\left[0, T_{0}\right]} E\left|\varepsilon^{h}(t)\right|=0, \quad \text { for any } 0<T_{0}<\infty .
$$

The modified dynamics of (9) corresponding to (36) is given by

$$
\begin{aligned}
\xi(t) & =x_{0}+\int_{0}^{t} \bar{b}(\xi(s), \varphi(t)) d s \\
& +\int_{0}^{t} \bar{\sigma}(\xi(s), \varphi(t)) d w(s)-z(t)-g(t),
\end{aligned}
$$

with the presence of the reflection component $g(\cdot)$ and the harvesting component $z(\cdot)$. The corresponding cost function and value function are given by

$$
\begin{aligned}
& J\left(x_{0}, \varphi_{0}, z(\cdot)\right)=\sum_{i=1}^{r} E_{x_{0}, \varphi_{0}} \int_{0}^{\tau} e^{-a s} \bar{f}^{i}(\varphi(s)) d z^{i}(s), \\
& V\left(x_{0}, \varphi_{0}\right)=\sup _{z(\cdot)} J\left(x_{0}, \varphi_{0}, z(\cdot)\right) .
\end{aligned}
$$

Time rescaling. Our ultimate goal is to show that $V^{h}$ converges to $V$ in a large enough interval $[0, U]$ as $h=\left(h_{1}, h_{2}\right) \rightarrow(0,0)$. A common approach for proving the convergence of $V^{h}$ to $V$ is to begin by showing that the collection $\left\{\xi^{h}(\cdot), \psi^{h}(\cdot), w^{h}(\cdot), g^{h}(\cdot), z^{h}(\cdot)\right\}$ is tight, and then characterize the subsequential weak limits suitably. However, showing the tightness of the above family becomes problematic since in general, the family $\left\{g^{h}(\cdot), z^{h}(\cdot)\right\}$ may fail to be tight. To overcome this difficulty, the analysis must be done in a "stretched-out" time scale. First the rescaled time increments $\left\{\Delta \widehat{t}_{n}^{h}\right.$ : $n=0,1, \ldots\}$ are defined as follows

$$
\begin{aligned}
& \Delta \widehat{t}_{n}^{h}=h_{2} \mathbb{1}_{\left\{\pi_{n}^{h}=0\right\}}+h_{1} \mathbb{1}_{\left\{\pi_{n}^{h} \leq-1\right\}}+h_{1} \mathbb{1}_{\left\{\pi_{n}^{h} \geq 1\right\}}, \\
& \widehat{t}_{0}=0, \quad \widehat{t}_{n}=\sum_{k=0}^{n-1} \Delta \widehat{t}_{k}^{h}, \quad n \geq 1 .
\end{aligned}
$$

The time scale is stretched out by $h_{1}$ at the reflection and harvesting steps.

Definition 1 The rescaled time process $\widehat{T}^{h}(\cdot)$ is the unique continuous nondecreasing process satisfying (a) $\widehat{T}^{h}(0)=0$; (b) the derivative of $\widehat{T}^{h}(\cdot)$ is 1 on $\left(\widehat{t}_{n}^{h}, \widehat{t}_{n+1}^{h}\right)$ if $\pi_{n}^{h}=0$, i.e., $n$ is a diffusion step; (c) the derivative of $\widehat{T}^{h}(\cdot)$ is 0 on $\left(\widehat{t}_{n}^{h}, \widehat{t}_{n+1}^{h}\right)$ if $\pi_{n}^{h} \neq 0$, i.e., $n$ is a reflection step or a harvesting step.

Thus $\widehat{T}^{h}(\cdot)$ does not increase at these $t$ at which a harvesting step or a reflection step occurs. It follows from the above definition that $\widehat{T}^{h}\left(\widehat{t}_{n}\right)=t_{n}^{h}$ and $\widehat{T}^{h}\left(\widehat{t}_{n+1}\right)-$
$\widehat{T}^{h}\left(\widehat{t}_{n}\right)=\Delta t_{n}^{h}$. Moreover, for $t \geq 0$ and $\delta>0,0 \leq$ $\widehat{T}^{h}(t+\delta)-\widehat{T}^{h}(t) \leq \delta$. Define the rescaled and interpolated process $\widehat{\xi}^{h}(t)=\xi^{h}\left(\widehat{T}^{h}(t)\right)$ and likewise define $\widehat{\psi}^{h}(\cdot), \widehat{z}^{h}(\cdot), \widehat{g}^{h}(\cdot), \widehat{B}^{h}(\cdot), \widehat{M}^{h}(\cdot)$, and the filtration $\widehat{\mathcal{F}}^{h}(\cdot)$ similarly. It follows from (32) that

$$
\widehat{\xi}^{h}(t)=x_{0}+\widehat{B}^{h}(t)+\widehat{M}^{h}(t)-\widehat{z}^{h}(t)-\widehat{g}^{h}(t) .
$$

Using the same argument that produced (36) we obtain

$$
\begin{aligned}
& \widehat{\xi}^{h}(t)=x_{0}+\int_{0}^{t} \bar{b}\left(\widehat{\xi}^{h}(s), \widehat{\psi}^{h}(s)\right) d \widehat{T}^{h}(s) \\
& +\int_{0}^{t} \bar{\sigma}\left(\widehat{\xi}^{h}(s), \widehat{\psi}^{h}(s)\right) d \widehat{w}^{h}(s)-\widehat{z}^{h}(t)-\widehat{g}^{h}(t)+\widehat{\varepsilon}^{h}(t),
\end{aligned}
$$

with $\widehat{\varepsilon}^{h}(\cdot)$ is an $\widehat{\mathcal{F}}^{h}(\cdot)$-adapted process satisfying

$$
\lim _{h \rightarrow 0} \sup _{t \in\left[0, T_{0}\right]} E\left|\widehat{\varepsilon}^{h}(t)\right|=0, \quad \text { for any } 0<T_{0}<\infty \text {. }
$$

Denote $\widehat{H}^{h}(\cdot)=\left(\widehat{\xi}^{h}(\cdot), \widehat{\psi}^{h}(\cdot), \widehat{w}^{h}(\cdot), \widehat{z}^{h}(\cdot), \widehat{g}^{h}(\cdot), \widehat{T}^{h}(\cdot)\right)$. To proceed, we give the definition of existence and uniqueness of weak solution and state some more assumptions.

Definition 2 By a weak solution of (6)-(37) we mean that there exists a probability space $(\Omega, \mathcal{F}, P)$, a filtration $\mathcal{F}(t)$, and process $(\xi(\cdot), \psi(\cdot), z(\cdot), g(\cdot), w(\cdot), \bar{w}(\cdot))$ such that $w(\cdot)$ and $\bar{w}(\cdot)$ are independent $\mathcal{F}(t)$-Wiener processes, $z(\cdot)$ and $g(\cdot)$ are $\mathcal{F}(t)$-adapted, and (6)(37) are satisfied. For an initial condition $\left(x_{0}, \varphi_{0}\right)$, by the weak sense uniqueness, we mean that irrespective of probability space, the probability law of solution $(\xi(\cdot), \psi(\cdot), z(\cdot), g(\cdot), w(\cdot), \bar{w}(\cdot))$ to $(6)-(37)$ is determined by the probability law of $(\psi(\cdot), z(\cdot), w(\cdot), \bar{w}(\cdot))$.

(A4) For each initial condition, there exists a solution to (6)-(37) and this solution is unique in the weak sense.

\subsection{Proof of Convergence}

In this subsection, we use the weak convergence methods to obtain the convergence of the algorithms. We refer the readers to Billingsley (1968), Ethier and Kurtz (1986) for standard references. Let $D[0, \infty)$ denote the space of functions that are right continuous and have left-hand limits endowed with the Skorohod topology. All the weak analysis will be on this space or its $k$-fold products $D^{k}[0, \infty)$ for appropriate $k$.

Theorem 3 Assume (A1)-(A4). Let the approximating chain $\left\{\xi_{n}^{h}\right\}$ be constructed with transition probabilities defined in (24)-(28), $H^{h}(\cdot)=\left(\xi^{h}(\cdot), \psi^{h}(\cdot), w^{h}(\cdot), z^{h}(\cdot)\right.$, $\left.g^{h}(\cdot), T^{h}(\cdot)\right)$ be the continuous-time interpolation defined in (29)-(30), (34), Definition 1, and $\widehat{H}^{h}(\cdot)$ be the corresponding rescaled processes. Then $\widehat{H}^{h}(\cdot)$ is tight. As a result, $\widehat{H}^{h}(\cdot)$ has a weakly convergent subsequence with the limit denoted by $\widehat{H}(\cdot)=(\widehat{\xi}(\cdot), \widehat{\psi}(\cdot), \widehat{w}(\cdot), \widehat{z}(\cdot), \widehat{g}(\cdot), \widehat{T}(\cdot))$, having continuous paths w.p.1. 
Proof. It follows from the definition of $\left\{\psi_{n}^{h}\right\}$ and interpolation intervals constructed in (21) that if $n$ is a harvesting step or a reflection step, $\Delta t_{n}^{h}=t_{n+1}^{h}-t_{n}^{h}=0$ and $\psi_{n+1}^{h}=\psi_{n}^{h}$. Otherwise, $n$ is a diffusion step, $\Delta t_{n}^{h}=$ $t_{n+1}^{h}-t_{n}^{h}=h_{2}$ and $\psi_{n+1}^{h}=\varphi_{k+1}^{h}$ if $\psi_{n}^{h}=\varphi_{k}^{h}$. By virtue of the continuous time interpolation $\psi^{h}(\cdot)$ in (30), we have $\psi^{h}(t)=\varphi_{k}^{h}$, for $t \in\left[k h_{2}, k h_{2}+h_{2}\right)$ and $k=0,1, \ldots$, with the sequence $\left\{\varphi_{n}^{h}\right\}$ constructed in (18). With this observation, the tightness of $\psi^{h}(\cdot)$ can be obtained as in Yin and Zhang (2006) (Theorem 8.15). For other components, we use the same estimations as in Kushner and Martins (1991) (Theorem 5.3) using the tightness criteria in Kushner (1984) (p. 47). Let $T_{0}<\infty$ be a positive constant and $\tau_{0}$ be a stopping time which is not bigger than $T_{0}$. Then for any $\delta>0$,

$$
E_{\tau_{0}}^{h}\left|w^{h}\left(\tau_{0}+\delta\right)-w^{h}\left(\tau_{0}\right)\right|^{2}=O(\delta)+\varepsilon^{h}(\delta),
$$

where terms $E\left|\varepsilon^{h}(\delta)\right| \rightarrow 0$ uniformly in $\tau_{0}$ as $h \rightarrow 0$. Taking $\lim \sup _{h \rightarrow 0}$ followed by $\lim _{\delta \rightarrow 0}$ yield the tightness of $\left\{w^{h}(\cdot)\right\}$. The tightness of $\left\{\widehat{\psi}^{h}(\cdot)\right\}$ and $\left\{\widehat{w}^{h}(\cdot)\right\}$ are obtained due to the stretching out of the timescale. Using the definition of "stretched out" timescale,

$$
\begin{aligned}
& E_{\tau_{0}}^{h}\left|\widehat{z}^{h}\left(\tau_{0}+\delta\right)-\widehat{z}^{h}\left(\tau_{0}\right)\right|^{2} \\
& \leq r h_{1}^{2} E_{\tau_{0}}^{h}(\text { number of harvesting steps in } \\
& \left.\quad \text { interpolated interval }\left[\tau_{0}, \tau_{0}+\delta\right)\right)^{2} \\
& \leq r h_{1}^{2} \max \left\{1, \delta^{2} / h_{1}^{2}\right\} \leq r\left(h_{1}^{2}+\delta^{2}\right) .
\end{aligned}
$$

Similarly,

$$
E_{\tau_{0}}^{h}\left|\widehat{g}^{h}\left(\tau_{0}+\delta\right)-\widehat{g}^{h}\left(\tau_{0}\right)\right|^{2} \leq r\left(h_{1}^{2}+\delta^{2}\right) .
$$

Thus $\left\{\widehat{z}^{h}(\cdot), \widehat{g}^{h}(\cdot)\right\}$ is tight. The tightness of $\left\{\widehat{T}^{h}(\cdot)\right\}$ follows from the fact that $0 \leq \widehat{T}^{h}\left(\tau_{0}+\delta\right)-\widehat{T}^{h}\left(\tau_{0}\right) \leq \delta$. Next we prove the tightness of $\left\{\widehat{\xi}^{h}(\cdot)\right\}$. It follows from (40), (44), and (45) that

$$
\begin{aligned}
& E_{\tau_{0}}^{h}\left|\widehat{\xi}^{h}\left(\tau_{0}+\delta\right)-\widehat{\xi}^{h}\left(\tau_{0}\right)\right|^{2} \leq 4 E_{\tau_{0}}^{h}\left|\widehat{B}^{h}\left(\tau_{0}+\delta\right)-\widehat{B}^{h}\left(\tau_{0}\right)\right|^{2} \\
& +4 E_{\tau_{0}}^{h}\left|\widehat{M}^{h}\left(\tau_{0}+\delta\right)-\widehat{M}^{h}\left(\tau_{0}\right)\right|^{2} \\
& +4 E_{\tau_{0}}^{h}\left|\widehat{z}^{h}\left(\tau_{0}+\delta\right)-\widehat{z}^{h}\left(\tau_{0}\right)\right|^{2}+4 E_{\tau_{0}}^{h}\left|\widehat{g}^{h}\left(\tau_{0}+\delta\right)-\widehat{g}^{h}\left(\tau_{0}\right)\right|^{2} \\
& \leq K \delta^{2}+K \delta+8 r\left(h_{1}^{2}+\delta^{2}\right),
\end{aligned}
$$

where $K$ is a positive constant depending only on upper bounds of $\bar{b}(\cdot, \cdot)$ and $\bar{\sigma}(\cdot, \cdot)$ on $L_{h_{1}} \times S_{m-1} \subset[0, U+1]^{r} \times$ $S_{m-1}$. This show the tightness of $\left\{\widehat{\xi}^{h}(\cdot)\right\}$. Hence $\widehat{H}^{h}(\cdot)$ is tight. By virtue of Prohorov's Theorem, $\widehat{H}^{h}(\cdot)$ has a weakly convergent subsequence with the limit $\widehat{H}(\cdot)$.

By the definition of $\widehat{T}^{h}(\cdot)$, it is Lipschitz continuous with Lipschitz coefficient 1. By virtue of the Skorohod representation, such property also holds for $\widehat{T}(\cdot)$. Since sizes of jumps of $\widehat{\xi}^{h}(\cdot), \widehat{w}^{h}(\cdot), \widehat{z}^{h}(\cdot)$, and $\widehat{g}^{h}(\cdot)$ go to 0 as $h \rightarrow 0$, then their limits have continuous paths w.p.1; see Kushner (1990) (p. 1007). Finally, consider the tight sequence $\left(\psi^{h}(\cdot), \widehat{\psi}^{h}(\cdot), \widehat{T}^{h}(\cdot)\right)$ with the weak limit $(\widetilde{\psi}(\cdot), \widehat{\psi}(\cdot), \widehat{T}(\cdot))$. Using the same argument as in Yin and Zhang (2006) (Section 8.4), we obtain that $\widetilde{\psi}(\cdot)$ solves the Wonham filter equation, then it has continuous paths w.p.1. It then follows from $\widehat{\psi}^{h}(\cdot)=\psi^{h}\left(\widehat{T}^{h}(\cdot)\right)$ that $\widehat{\psi}(\cdot)=\widetilde{\psi}(\widehat{T}(\cdot))$. Therefore, $\widehat{\psi}(\cdot)$ has also continuous paths w.p.1. This completes the proof. $\square$ In what follows, for notational simplicity, we still denote the convergent subsequence of $\widehat{H}^{h}(\cdot)$ by $\widehat{H}^{h}(\cdot)$. By Skorohod's representation, with a slight abuse of notation, we can always assume that the convergence is also pathwise w.p.1 in the topology of the path space and is uniform on bounded time interval. We proceed to characterize the limit process.

Theorem 4 Under conditions of Theorem 3, let $\widehat{\mathcal{F}}(t)$ be the $\sigma$-algebra generated by $\{\widehat{\xi}(s), \widehat{\psi}(s), \widehat{w}(s), \widehat{z}(s), \widehat{g}(s), \widehat{T}(s)$ : $s \leq t\}$. Then the following assertions hold. (a) $\widehat{w}(t)$ is an $\widehat{\mathcal{F}}(t)$ - martingale with quadratic variation process $\widehat{T}(t) I_{n} \cdot(\mathrm{b}) \widehat{z}(\cdot), \widehat{g}(\cdot)$, and $\widehat{T}(\cdot)$ are nondecreasing and nonnegative. (c) The limit processes satisfy

$$
\begin{aligned}
& \widehat{\xi}(t)=x_{0}+\int_{0}^{t} \bar{b}(\widehat{\xi}(s), \widehat{\psi}(s)) d \widehat{T}(s) \\
& +\int_{0}^{t} \bar{\sigma}(\widehat{\xi}(s), \widehat{\psi}(s)) d \widehat{w}(s)-\widehat{z}(t)-\widehat{g}(t) .
\end{aligned}
$$

Proof. (a) Let $\widehat{E}_{t}^{h}$ denote the expectation conditioned on $\widehat{\mathcal{F}}^{h}(t)=\mathcal{F}^{h}\left(\widehat{T}^{h}(t)\right)$. Recall that $w^{h}(\cdot)$ is an $\mathcal{F}^{h}(\cdot)$ martingale and by the definition of $\widehat{w}^{h}(\cdot)$, for any $\delta>0$,

$$
\begin{aligned}
& \widehat{E}_{t}^{h}\left(\widehat{w}^{h}(t+\delta)-\widehat{w}^{h}(t)\right)=0, \\
& \widehat{E}_{t}^{h}\left(\widehat{w}^{h}(t+\delta) \widehat{w}^{h}(t+\delta)^{\prime}-\widehat{w}^{h}(t) \widehat{w}^{h}(t)^{\prime}\right) \\
& =\left(\widehat{T}^{h}(t+\delta)-\widehat{T}^{h}(t)\right) I_{r}+\widehat{\varepsilon}^{h}(\delta),
\end{aligned}
$$

where $E\left|\widehat{\varepsilon}^{h}(\delta)\right| \rightarrow 0$ as $h \rightarrow 0$. To characterize $\widehat{w}(\cdot)$, let $q$ be an arbitrary integer, $t>0, \delta>0$ and $\left\{t_{k}: k \leq q\right\}$ be such that $t_{k} \leq t<t+\delta$ for each $k$. Let $\Psi(\cdot)$ be a realvalued and continuous function of its arguments with compact support. Then in view of (47), we have

$$
E \Psi\left(\widehat{H}^{h}\left(t_{k}\right), k \leq q\right)\left[\widehat{w}^{h}(t+\delta)-\widehat{w}^{h}(t)\right]=0,
$$

$$
\begin{gathered}
E \Psi\left(\widehat{H}^{h}\left(t_{k}\right), k \leq q\right)\left[\left(\widehat{w}^{h}(t+\delta) \widehat{w}^{h}(t+\delta)^{\prime}-\widehat{w}^{h}(t) \widehat{w}^{h}(t)^{\prime}\right.\right. \\
\left.-\left(\widehat{T}^{h}(t+\delta)-\widehat{T}^{h}(t)\right) I_{r}-\widehat{\varepsilon}^{h}(\delta)\right]=0 .
\end{gathered}
$$

By using the Skorohod representation and the dominated convergence theorem, letting $h \rightarrow 0$ in (48), we obtain

$$
E \Psi\left(\widehat{H}\left(t_{k}\right), k \leq q\right)[\widehat{w}(t+\delta)-\widehat{w}(t)]=0 .
$$

Since $\widehat{w}^{h}(\cdot)$ has continuous paths w.p.1, (50) implies that $\widehat{w}(\cdot)$ is a continuous $\widehat{\mathcal{F}}(\cdot)$-martingale. Moreover, (49) 
gives us that

$$
\begin{aligned}
& E \Psi\left(\widehat{H}\left(t_{k}\right), k \leq q\right)\left[\widehat{w}(t+\delta) \widehat{w}(t+\delta)^{\prime}-\widehat{w}(t) \widehat{w}(t)^{\prime}\right. \\
& \left.\quad-(\widehat{T}(t+\delta)-\widehat{T}(t)) I_{r}\right]=0 .
\end{aligned}
$$

Then part (a) follows.

(b) The monotonicity and non-negativity of $\widehat{z}(\cdot), \widehat{g}(\cdot)$, and $\widehat{T}(\cdot)$ follow immediately from that of $\widehat{z}^{h}(\cdot), \widehat{g}^{h}(\cdot)$, and $\widehat{T}^{h}(\cdot)$, respectively.

(c) The proof of this part is motivated by that of Kushner and Dupuis (1992) (Theorem 10.4.1). By virtue of Skorohod representation, the uniform convergence of $\left(\widehat{\xi}^{h}(\cdot), \widehat{\psi}^{h}(\cdot), \widehat{T}^{h}(\cdot)\right)$ to $(\widehat{\xi}(\cdot), \widehat{\psi}(\cdot), \widehat{T}(\cdot))$ on bounded time interval, we obtain that as $h \rightarrow 0$,

$$
\int_{0}^{t} \bar{b}\left(\widehat{\xi}^{h}(s), \widehat{\psi}^{h}(s)\right) d \widehat{T}^{h}(s)-\int_{0}^{t} \bar{b}(\widehat{\xi}(s), \widehat{\psi}(s)) d \widehat{T}(s) \rightarrow 0,
$$

uniformly in $t$ on any bounded time interval w.p.1. For each positive constant $\delta$ and a process $\widehat{\nu}(\cdot)$, define the piecewise constant process $\widehat{\nu}^{\delta}(\cdot)$ by $\widehat{\nu}^{\delta}(t)=\widehat{\nu}(k \delta)$ for $t \in[k \delta, k \delta+\delta), k=0,1, \ldots$ Then, by the tightness of $\left(\widehat{\xi}^{h}(\cdot), \widehat{\psi}^{h}(\cdot)\right),(41)$ can be rewritten as

$$
\begin{aligned}
& \widehat{\xi}^{h}(t)=x_{0}+\int_{0}^{t} \bar{b}\left(\widehat{\xi}^{h}(s), \widehat{\psi}^{h}(s)\right) d \widehat{T}^{h}(s) \\
& +\int_{0}^{t} \bar{\sigma}\left(\widehat{\xi}^{h, \delta}(s), \widehat{\psi}^{h, \delta}(s)\right) d \widehat{w}^{h}(s)-\widehat{z}^{h}(t)-\widehat{g}^{h}(t)+\widehat{\varepsilon}^{h, \delta}(t),
\end{aligned}
$$

where $\lim _{\delta \rightarrow 0} \lim \sup _{h \rightarrow 0} E\left|\widehat{\varepsilon}^{h, \delta}(t)\right|=0$. Owing to the fact that $\widehat{\xi}^{h, \delta}$ and $\widehat{\psi}^{h, \delta}$ take constant values on the intervals $[n \delta, n \delta+\delta)$, we have

$\int_{0}^{t} \bar{\sigma}\left(\widehat{\xi}^{h, \delta}(s), \widehat{\psi}^{h, \delta}(t)\right) d \widehat{w}^{h}(s) \rightarrow \int_{0}^{t} \bar{\sigma}\left(\widehat{\xi}^{\delta}(s), \widehat{\psi}^{\delta}(t)\right) d \widehat{w}(s)$,

as $h \rightarrow 0$, which are well defined w.p. 1 since they can be written as finite sums. Combining (51)-(53), we have

$$
\begin{aligned}
& \widehat{\xi}(t)=x_{0}+\int_{0}^{t} \bar{b}(\widehat{\xi}(s), \widehat{\psi}(s)) d \widehat{T}(s) \\
& +\int_{0}^{t} \bar{\sigma}\left(\widehat{\xi}^{\delta}(s), \widehat{\psi}^{\delta}(t)\right) d \widehat{w}(s)-\widehat{z}(t)-\widehat{g}(t)+\widehat{\varepsilon}^{\delta}(t),
\end{aligned}
$$

where $\lim _{\delta \rightarrow 0} E\left|\widehat{\varepsilon}^{\delta}(t)\right|=0$. Taking limit in the above equation as $\delta \rightarrow 0$ yields the result.

Theorem 5 Under conditions of Theorem 3, for $t<\infty$, define the reverse $R(t)=\inf \{s: \widehat{T}(s)>t\}$. For any process $\widehat{\nu}(\cdot)$, define the rescaled process $\nu(\cdot)$ by $\nu(t)=\widehat{\nu}(R(t))$. Let $\mathcal{F}(t)$ be the $\sigma$-algebra generated by $\{\xi(s), \psi(s), w(s), z(s), g(s), R(s): s \leq t\}$. The following assertions hold: (a) $R(\cdot)$ is right continuous, nondecreasing, and $R(t) \rightarrow \infty$ as $t \rightarrow \infty$ w.p.1. (b) $z(\cdot)$ and $g(\cdot)$ are right-continuous, nondecreasing, nonnegative, and $\mathcal{F}(t)$-adapted processes. (c) $w(\cdot)$ is a standard $\mathcal{F}(t)$ Wiener process, $\psi(\cdot)$ satisfies the system of Wonham filter equations (5), and (37) holds.
Proof. (a) We will argue via contradiction that $\widehat{T}(t) \rightarrow$ $\infty$ as $t \rightarrow \infty$ w.p.1. Suppose $P\left[\sup _{t \geq 0} \widehat{T}(t)<\infty\right]>0$. Then there exist positive constants $\varepsilon$ and $T_{0}$ such that

$$
P\left[\sup _{t \geq 0} \widehat{T}(t)<T_{0}-1\right]>\varepsilon .
$$

We first observe that

$$
\begin{aligned}
& t+r\left|z^{h}(t)+g^{h}(t)\right| \\
& \geq \sum_{k=0}^{n^{h}(t)-1}\left(h_{2} \mathbb{1}_{\left\{\pi^{h}=0\right\}}+h_{1} \mathbb{1}_{\left\{\pi^{h} \geq 1\right\}}+h_{1} \mathbb{1}_{\left\{\pi^{h} \leq-1\right\}}\right) .
\end{aligned}
$$

Since $\widehat{T}^{h}(\cdot)$ is nondecreasing and $\widehat{T}^{h}\left(\widehat{t}_{n}^{h}\right)=t_{n}^{h}$,

$$
\begin{aligned}
& \widehat{T}^{h}\left(t+r\left|z^{h}(t)+g^{h}(t)\right|\right) \\
\geq & \widehat{T}^{h}\left(\sum_{k=0}^{n^{h}(t)-1}\left(h_{2} \mathbb{1}_{\left\{\pi^{h}=0\right\}}+h_{1} \mathbb{1}_{\left\{\pi^{h} \geq 1\right\}}+h_{1} \mathbb{1}_{\left\{\pi^{h} \leq-1\right\}}\right)\right) \\
= & \widehat{T}^{h}\left(\widehat{t}_{n^{h}(t)}^{h}\right)=t_{n^{h}(t)}^{h} \geq t-1 .
\end{aligned}
$$

The last inequality above is a consequence of the inequalities $t_{n^{h}(t)}^{h} \leq t<t_{n^{h}(t)+1}^{h}=t_{n^{h}(t)}^{h}+h_{2}<t_{n^{h}(t)}^{h}+1$.

It follows from (32) that for each fixed $t \geq 0$, $\sup _{h} E\left(\left|z^{h}(t)+g^{h}(t)\right|\right)<\infty$. Hence for a sufficiently large $K$,

$P\left\{r\left|z^{h}\left(T_{0}\right)+g^{h}\left(T_{0}\right)\right| \geq 2 K\right\} \leq \frac{r E\left|z^{h}\left(T_{0}\right)+g^{h}\left(T_{0}\right)\right|}{2 K}<\frac{\varepsilon}{2}$.

In view of (55) and (56), we obtain

$$
\begin{aligned}
& P\left[\widehat{T}^{h}\left(T_{0}+2 K\right)<T_{0}-1\right] \\
& \leq P\left[\widehat{T}^{h}\left(T_{0}+r\left|z^{h}\left(T_{0}\right)+g^{h}\left(T_{0}\right)\right|\right)<T_{0}-1, r \mid z^{h}\left(T_{0}\right)\right. \\
& \left.+g^{h}\left(T_{0}\right) \mid<2 K\right]+P\left[r\left|z^{h}\left(T_{0}\right)+g^{h}\left(T_{0}\right)\right| \geq 2 K\right] \\
& <\frac{\varepsilon}{2}, \quad \text { for all small } h=\left(h_{1}, h_{2}\right) .
\end{aligned}
$$

Since $\widehat{T}^{h}$ converges weakly to $\widehat{T}$, it follows from (57) that $\liminf _{h \rightarrow 0} P\left[\widehat{T}^{h}\left(T_{0}+2 K\right)<T_{0}-1\right] \leq \varepsilon / 2$. This contradicts (54); see Billingsley (1968) (Theorem 1.2.1). Hence $\widehat{T}(t) \rightarrow \infty$ as $t \rightarrow \infty$ w.p.1. Thus $R(t)<\infty$ for all $t$ and $R(t) \rightarrow \infty$ as $t \rightarrow \infty$. Since $\widehat{T}(\cdot)$ is nondecreasing and continuous, $R(\cdot)$ is nondecreasing and right-continuous. (b) follows the fact that $\widehat{z}(\cdot)$ and $\widehat{g}(\cdot)$ are continuous, nondecreasing, nonnegative, and $R(\cdot)$ is right-continuous.

(c) We first note that although $R(\cdot)$ might fail to be continuous, $w(\cdot)=\widehat{w}(R(\cdot))$ has continuous paths w.p.1. Indeed, consider the tight sequence $\left(w^{h}(\cdot), \widehat{w}^{h}(\cdot), \widehat{T}^{h}(\cdot)\right)$ with the weak limit $(\widetilde{w}(\cdot), \widehat{w}(\cdot), \widehat{T}(\cdot))$. Since $\widehat{w}^{h}(\cdot)=$ $w^{h}\left(\widehat{T}^{h}(\cdot)\right)$, we must have that $\widehat{w}(\cdot)=\widetilde{w}(\widehat{T}(\cdot))$. It follows from the definition of $R(\cdot)$ that for each $t \geq 0$, we have $\widehat{T}(R(t))=t$. Hence $w(t)=\widehat{w}(R(t))=\widetilde{w}(\widehat{T}(R(t)))=$ $\widetilde{w}(t)$. Since size of jumps of $w^{h}(\cdot)$ go to 0 as $h \rightarrow 0$, $\widetilde{w}(\cdot)$ also has continuous paths w.p.1. This shows that 
$w(\cdot)=\widehat{w}(R(\cdot))$ has continuous paths w.p.1. By the same argument for the tight sequence $\left(\psi^{h}(\cdot), \widehat{\psi}^{h}(\cdot), \widehat{T}^{h}(\cdot)\right)$, we obtain that $\psi(\cdot)$ also has continuous paths w.p.1. Moreover, it satisfies the system of Wonham filter equation (5); see Yin and Zhang (2006) (Section 8.4).

Before characterizing $w(\cdot)$, we note that for $t \geq 0$, $\{R(s) \leq t\}=\{\widehat{T}(t) \geq s\} \in \widehat{\mathcal{F}}(t)$ since $\widehat{T}(t)$ is $\widehat{\widehat{\mathcal{F}}}(t)$ measurable. Thus $R(s)$ is an $\widehat{\mathcal{F}}(t)$-stopping time for each $s \geq 0$. Since $\widehat{w}(t)$ is an $\widehat{\mathcal{F}}(t)$-martingale with quadratic variation process $\widehat{T}(t) I_{n}$,

$E[\widehat{w}(R(t) \wedge n) \mid \widehat{\mathcal{F}}(R(s))]=\widehat{w}(R(s) \wedge n), \quad n=1,2, \ldots$, $E \widehat{w}(R(t) \wedge n) \widehat{w}(R(t) \wedge n)^{\prime}=E \widehat{T}(R(t) \wedge n) I_{r}$,

and $\widehat{T}(R(t) \wedge n) \leq \widehat{T}(R(t))=t$. Hence for each fixed $t \geq 0$, the family $\{\widehat{w}(R(t) \wedge n), n \geq 1\}$ is uniformly integrable. By that uniform integrability, we obtain from (58) that $E[\widehat{w}(R(t)) \mid \widehat{\mathcal{F}}(R(s))]=\widehat{w}(R(s))$, that is $E[w(t) \mid \mathcal{F}(s)]=w(s)$. This proves that $w(\cdot)$ is a continuous $\mathcal{F}(\cdot)$-martingale. We next consider its quadratic variation. By the Burkholder-Davis-Gundy inequality, there exists a positive constant $C$ independent of $n=1,2, \ldots$ such that

$$
\begin{aligned}
& E|\widehat{w}(R(t) \wedge n)|^{2} \leq C E\left[\left(\sup _{0 \leq s \leq R(t)}|\widehat{w}(R(s) \wedge n)|^{2}\right)\right] \\
& \leq C E|\widehat{T}(R(t) \wedge n)| \leq C t .
\end{aligned}
$$

Thus the families $\{\widehat{w}(R(t) \wedge n), n \geq 1\}$ and $\{\widehat{T}(R(t) \wedge$ $n), n \geq 1\}$ are uniformly integrable for each fixed $t \geq 0$. Combining with the fact that $\widehat{w}(\cdot), \widehat{T}(\cdot)$ have continuous paths, for nonnegative constants $s \leq t$, we have

$$
\begin{aligned}
& \widehat{w}(R(s) \wedge n) \widehat{w}(R(s) \wedge n)^{\prime}-\widehat{T}(R(s) \wedge n) I_{r} \\
& =E\left[\widehat{w}(R(t) \wedge n) \widehat{w}(R(t) \wedge n)^{\prime}-\widehat{T}(R(t) \wedge n) I_{r} \mid \widehat{\mathcal{F}}(R(s))\right] \\
& \rightarrow E\left[\widehat{w}(R(t)) \widehat{w}(R(t))^{\prime}-\widehat{T}(R(s)) I_{r} \mid \widehat{\mathcal{F}}(R(s))\right] \\
& =E\left[w(t) w(t)^{\prime}-t I_{r} \mid \mathcal{F}(s)\right] .
\end{aligned}
$$

Note that the first equation in (59) follows from the martingale property of $\widehat{w}(\cdot) \widehat{w}(\cdot)^{\prime}-\widehat{T}(\cdot) I_{r}$ with respect to $\widehat{\mathcal{F}}(t)$. Letting $n \rightarrow \infty$ in $(59)$, we arrive at $E\left[w(t) w(t)^{\prime}-\right.$ $\left.t I_{r} \mid \mathcal{F}(s)\right]=w(s) w(s)^{\prime}-s I_{r}$. Therefore, $w(\cdot)$ is an $\mathcal{F}(t)$ - Wiener process. A rescaling of (46) yields (37). The proof is complete.

Theorem 6 Under conditions of Theorem 3, let $V^{h}\left(x_{0}, \varphi_{0}\right)$ and $V\left(x_{0}, \varphi_{0}\right)$ be value functions defined in (23) and (38), respectively. Then $V^{h}\left(x_{0}, \varphi_{0}\right) \rightarrow$ $V\left(x_{0}, \varphi_{0}\right)$ as $h \rightarrow 0$.

Proof. We first show that as $h \rightarrow 0$,

$$
J^{h}\left(x_{0}, \varphi_{0}, z^{h}\right) \rightarrow J\left(x_{0}, \varphi_{0}, z(\cdot)\right) .
$$

Indeed, for a harvesting strategy $z^{h}=\left\{z_{n}^{h}\right\}$, we have

$$
\begin{aligned}
J^{h}\left(x_{0}, \varphi_{0}, z^{h}\right) & =\sum_{i=1}^{r} E \sum_{k=0}^{\eta_{h}-1} e^{-a t_{k}^{h}} \bar{f}^{i}\left(\psi_{k}^{h}\right) \Delta z_{k}^{h, i} \\
& =\sum_{i=1}^{r} E \int_{0}^{\widehat{t}_{\eta_{h}}^{h}} e^{-a \widehat{T}^{h}(t)} \bar{f}^{i}\left(\widehat{\psi}^{h}(t)\right) d \widehat{z}^{h, i}(t),
\end{aligned}
$$

where $\widehat{z}^{h}(\cdot)=\left(\widehat{z}^{h, 1}(\cdot), \ldots, \widehat{z}^{h, r}(\cdot)\right)^{\prime}$. By a small modification of the proof in Theorem 5 (a), we have $\widehat{T}^{h}(t) \rightarrow$ $\infty$ as $t \rightarrow \infty$ w.p.1. It also follows from the representation (32) and estimates on $B^{h}(\cdot)$ and $M^{h}(\cdot)$ that $\left\{z^{h}(n+1)-z^{h}(n): n, h\right\}$ is uniformly integrable. Thus, by the definition of $\widehat{T}^{h}(\cdot)$,

$$
\begin{aligned}
& \sum_{i=1}^{r} E \int_{T_{0}}^{\infty} e^{-a \widehat{T}^{h}(t)} \bar{f}^{i}\left(\widehat{\psi}^{h}(t)\right) d \widehat{z}^{h, i}(t) \\
& \leq \sum_{i=1}^{r} E \int_{\min \left\{t: \widehat{T}^{h}(t) \geq T_{0}\right\}}^{\infty} K e^{-a s} d z^{h, i}(s) \\
& \leq \sum_{i=1}^{r} E \int_{T_{0}}^{\infty} K e^{-a s} d z^{h, i}(s) \rightarrow 0, \quad T_{0} \rightarrow \infty,
\end{aligned}
$$

uniformly in $h$, where $z^{h}(\cdot)=\left(z^{h, 1}(\cdot), \ldots, z^{h, r}(\cdot)\right)^{\prime}$. In the above argument, we have employed the fact that we can replace $\widehat{t}_{\eta_{h}}^{h}$ in (61) by infinity, and $\widehat{T}^{h}\left(T_{0}\right) \leq T_{0}$. Then by the weak convergence, the Skohorod representation (hence the uniform convergence of $\left(\widehat{z}^{h}(\cdot), \widehat{\psi}^{h}(\cdot), \widehat{T}^{h}(\cdot)\right)$ to $(\widehat{z}(\cdot), \widehat{\psi}(\cdot), \widehat{T}(\cdot))$ on bounded time interval), and uniform integrability, for any $T_{0}>0$,

$$
\begin{aligned}
\sum_{i=1}^{r} E & \int_{0}^{T_{0}} e^{-a \widehat{T}^{h}(t)} \bar{f}^{i}\left(\widehat{\psi}^{h}(t)\right) d \widehat{z}^{h, i}(t) \\
& \rightarrow \sum_{i=1}^{r} E \int_{0}^{T_{0}} e^{-a \widehat{T}(t)} \bar{f}^{i}(\widehat{\psi}(t)) d \widehat{z}^{i}(t) .
\end{aligned}
$$

The above convergence holds when we replace $T_{0}$ by infinity, or equivalently,

$$
\begin{aligned}
& J^{h}\left(x_{0}, \varphi_{0}, z^{h}\right)=\sum_{i=1}^{r} E \int_{0}^{\widehat{t}_{\eta_{h}}^{h}} e^{-a \widehat{T}^{h}(t)} \bar{f}^{i}\left(\widehat{\psi}^{h}(t)\right) d \widehat{z}^{h, i}(t) \\
& \rightarrow \sum_{i=1}^{r} E \int_{0}^{\widehat{\tau}^{i=1}} e^{-a \widehat{T}(t)} \bar{f}^{i}(\widehat{\psi}(t)) d \widehat{z}^{i}(t),
\end{aligned}
$$

where $\widehat{\tau}=\inf \{s: \widehat{\xi}(s)=0\}$. On inversion of the timescale, the limit can be written as $\sum_{i=1}^{r} E \int_{0}^{\tau} e^{-a t}$ $\bar{f}^{i}(\psi(t)) d z^{i}(t)=J\left(x_{0}, \varphi_{0}, z(\cdot)\right)$. Thus, (60) follows. The rest of the proof is divided in two parts. First, using a standard argument as in Jin et al. (2012), we obtain $\lim \sup _{h} V^{h}\left(x_{0}, \varphi_{0}\right) \leq V\left(x_{0}, \varphi_{0}\right)$. Next, to prove the reverse inequality $\liminf _{h} V^{h}\left(x_{0}, \varphi_{0}\right) \geq V\left(x_{0}, \varphi_{0}\right)$, for any small positive constant $\varepsilon$, we choose a particular $\varepsilon$-optimal harvesting strategy for (6) and (37) such that the approximation can be applied to the chain $\left\{\xi_{n}^{h}\right\}$ and the associated cost compared with $V^{h}\left(x_{0}, \varphi_{0}\right)$, i.e., 
$V^{h}\left(x_{0}, \varphi_{0}\right) \geq V\left(x_{0}, \varphi_{0}\right)-\varepsilon$. We use an adaption of the method used for singular control problems Kushner and Martins (1991); Martins and Kushner (1990). We omit the details due to the page limit.

\subsection{A Numerical Example}

We consider a single species ecosystem in random environment subjected to the harvesting as follows

$d \xi(t)=\xi(t)(b(\alpha(t))-c(\alpha(t)) \xi(t))+\sigma(\alpha(t)) d w(t)-d Z(t)$.

Suppose that the Markov chain $\alpha(\cdot) \in\{1,2\}$ that models random environment. The generator of the continuoustime Markov chain is given by $Q=\left(\begin{array}{cc}-1 & 1 \\ 1 & -1\end{array}\right)$, and $b(1)=2, c(1)=2, \sigma(1)=1, b(2)=5, c(2)=1, \sigma(2)=$ 0.5 . We suppose that the Markov chain can only be observed through $d y(t)=g(\alpha(t)) d t+d B(t)$, where $g(1)=$ $-1, g(2)=1$. Using the Wonham filter, we can convert the incompletely observed system to the system with complete observation. Let $a=0.05, f(1)=f(2)=1$. In this case, $\varphi(t)=\varphi^{1}(t)$ and $\bar{f}(\varphi(t))=1$. Then for an admissible strategy $Z(\cdot), J(x, \varphi, Z)=E \int_{0}^{\tau} e^{-a s} d Z(s)$. Based on the algorithm constructed in Section 3, we carry out the computation by value iterations. Let $Z_{0}$ be the harvesting policy that drive the system to extinct immediately. Then $J\left(x, \varphi, Z_{0}\right)=x$ for all $(x, \varphi)$. Letting $Z_{0}$ be the initial harvesting strategy, we set the initial values $V_{0}^{h}(x, \varphi)=x, x=0, h_{1}, \ldots, U=10, \varphi=0, h_{1}, \ldots, 1$. For actual computations of $V(x, \varphi)$, we choose a positive number $U$ large enough and compute the value function in the bounded interval $[0, U]$. That treatment is effective since the population size $\xi(t)$ starting from a level $x_{0}$ cannot reach infinity. In the above, since the drift in (4.34) is negative for $\xi>5$, we take $U=10$. Numerical experiments show that choice is sufficiently large. We outline how to find an improved values of $V(\cdot, \cdot)$ as follows. At each level $x=h_{1}, 2 h_{1}, \ldots, U$, denote by $\pi(x, n)$ the action we choose, where $\pi(x, n)=2$ if we choose a harvesting action and $\pi(x, n)=1$ if we choose not to do such action. Thus, initially we choose $\pi(x, 0)=2$ for all $x$ and we seek for better policies which give larger values. If we take a harvesting action, an improved value of $V(x, \varphi)$ is calculated by using $V_{n+1}^{h, 2}(x, \varphi)=V_{n}^{h}\left(x-h_{1}, \varphi\right)+\bar{f}(\varphi) h_{1}$. Otherwise, we choose not to harvest. By plugging all the necessary finite difference expressions into the first part of system (16), we deduce that an improved value $V_{n+1}^{h, 1}(x, \varphi)$ is calculated by using

$$
\begin{aligned}
& V_{n+1}^{h, 1}(x, \varphi)=\frac{1}{1+a h_{2}}\left[V_{n}^{h}\left(x+h_{1}, \varphi\right) p^{h}\left(x, x+h_{1} \mid \varphi, \pi\right)\right. \\
& +V_{n}^{h}\left(x-h_{1}, \varphi\right) p^{h}\left(x, x-h_{1} \mid \varphi, \pi\right) \\
& +V_{n}^{h}\left(x, \varphi+h_{1}\right) \frac{\left(q^{11} \varphi+q^{21}-q^{21} \varphi\right)^{+} h_{2} h_{1}+\frac{1}{2} \varphi^{2}(-2+2 \varphi)^{2} h_{2}}{h_{1}} \\
& +V_{n}^{h}\left(x, \varphi-h_{1}\right) \frac{\left(q^{11} \varphi+q^{21}-q^{21} \varphi\right)^{-} h_{2} h_{1}+\frac{1}{2} \varphi^{2}(-2+2 \varphi)^{2} h_{2}}{h_{1}^{2}} \\
& +V_{n}^{h}(x, \varphi)\left(p^{h}(x, x \mid \varphi, \pi)\right. \\
& \left.\left.-\frac{\left|q^{11} \varphi+q^{21}-q^{21} \varphi\right| h_{2} h_{1}+\varphi^{2}(-2+2 \varphi)^{2} h_{2}}{h_{1}^{2}}\right)\right],
\end{aligned}
$$

where $\frac{1}{1+a h_{2}} \approx e^{-a h_{2}}$ plays the role of discounting part. Therefore, we can find the optimal action and the corresponding improved $V_{n+1}^{h}(x, \varphi)$ as follows: $\pi(x, n):=\operatorname{argmax}\left\{i=1,2: V_{n+1}^{h, i}(x, \varphi)\right\}$, $V_{n+1}^{h}(x, \varphi):=V_{n+1}^{h, \pi(x, n)}(x, \varphi)$. The iterations stop as soon as the increment $V_{n+1}^{h}(\cdot)-V_{n}^{h}(\cdot)$ is below some tolerance.

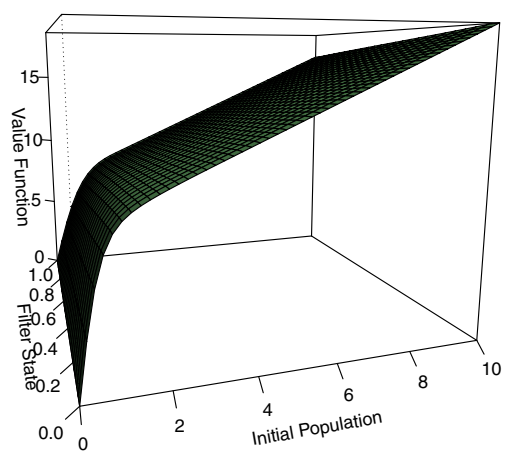

Fig. 1. Optimal value function versus initial population and initial filter state

Fig. 1 shows the value function $V(x, \varphi)$ as a function of initial population $x$ and initial filter state $\varphi$. To highlight the effect of initial filter states, in Fig. 2, we plot graphs of $V(\cdot, 0.1)$ and $V(\cdot, 0.9)$. Note that $J\left(\cdot, \cdot, Z_{0}\right)$ is also referred as current harvesting potential, and $V(\cdot, \cdot)-$ $J\left(\cdot, \cdot, Z_{0}\right)$ can be seen as the maximum present expected value of the accumulate net convenience yields accrued from postponing the harvesting decision and keeping the population alive after a small time interval; see Alvarez and Shepp (1998) and Tran and Yin (2015). Figure 3 and Figure 4 provide optimal policies, with " 1 " denoting not to harvest and "2" denoting harvesting actions. It can be seen from Fig. 1 and Fig. 2 that the value function is concave and the optimal harvesting policy is a barrier strategy. This notice agrees with observations and results in Jin et al. (2012) and Choulli et al. 


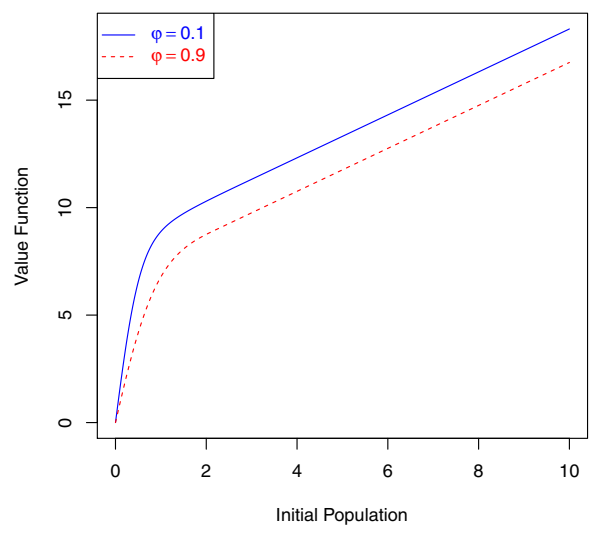

Fig. 2. Optimal value function versus initial population with $\varphi=0.1$ and $\varphi=0.9$

(2003). To be more specific, if the population is higher than some barrier level, a harvesting action is chosen, and the value function increases with unity slope (since we take $f(1)=f(2)=1$ ). Moreover, the barrier levels are different in different Wonham filter states.

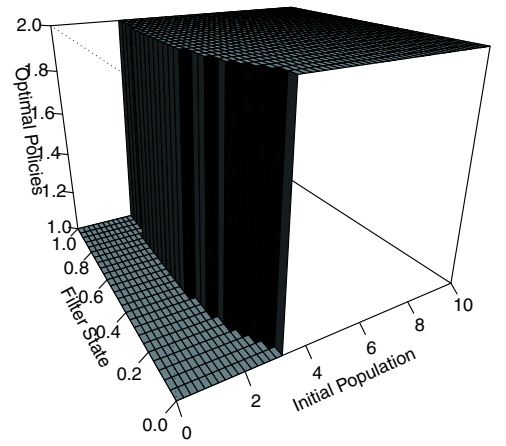

Fig. 3. Optimal policies versus initial population and initial filter state

\subsection{Further Remarks}

This paper focused on numerical methods for solution of optimal harvesting strategies in random environments. Note that different from applications in finance, in which the $X(t)$ is observed, here we have another observation $y(t)$ process. Such a model is natural for ecological systems. They also appear in many communication systems, networked systems, as well as cyber-physical systems. The novelties of our approach include that (1) we depicted the random environment as a hidden Markov chain; (2) we treated the resulting singular control problem under partial observations; (3) we used Wonham

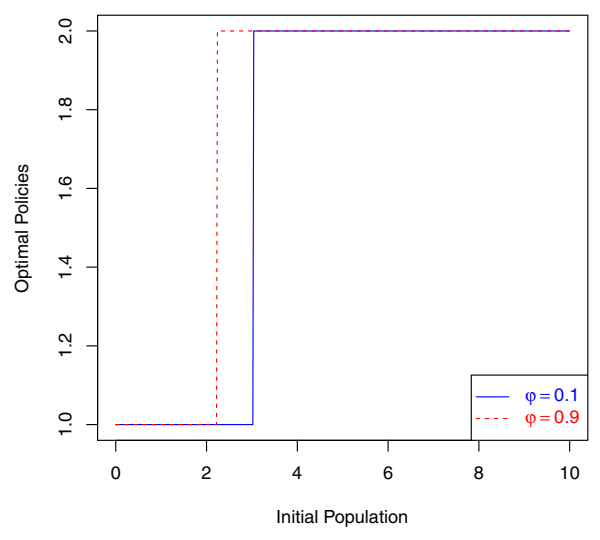

Fig. 4. Optimal policies versus initial population with $\varphi=0.1$ and $\varphi=0.9$

filter as a bridge to construct numerical approximation methods based on Markov chain approximation techniques to solve the optimal control problem under partial observations.

The convergence of the algorithms was proved. A numerical example was used to demonstrate the performance of our algorithm. The problem considered here can be modified to treat dividend optimization in insurance risk management, and networked control systems. Not only can the approach be applied the harvest problem under consideration, but also it opens up the domain for treating more general singular control problems under partial observations. Several directions may be worthwhile for further study and investigation. Using diffusions and switching diffusions with delays for modeling population dynamics has drawn much attention recently; see Liu and Bai (2015). A related problem of interest is to develop optimal harvesting strategies for such models. The Markov chain approximation method developed for numerical methods of controlled stochastic systems with delays in Kushner (2008) appears promising. Although for ecological applications, one usually examines continuous-time systems, it is also interesting to study problems in discrete time for other applications with a hidden Markov chain. A typical reference in this connection is Elliott, Aggoun, \& Moore (1995).

Acknowledgment. This research was supported in part by the Air Force Office of Scientific Research under FA9550-15-1-0131.

\section{References}

Alvarez, L. H., \& Shepp, L. A. (1998). Optimal harvesting of stochastically fluctuating populations. Journal of Mathematical Biology, 37(2), 155-177.

Asmussen, S., \& Taksar, M. (1997). Controlled diffusion models for optimal dividend pay-out. Insurance: Mathematics and Economics, 20(1), 1-15.

Billingsley, P. (1968). Convergence of probability measures. John Wiley \& Sons.

Budhiraja, A., \& Ross, K. (2007). Convergent numerical 
scheme for singular stochastic control with state constraints in a portfolio selection problem. SIAM Journal on Control and Optimization, 45(6), 2169-2206.

Elliott, R.J., Aggoun, L., \& Moore, J.B. (1995). Hidden Markov Models, Estimation and Control, Springer-Verlag, New York.

Ethier, S. N., \& Kurtz, T. G. (1986). Markov processes: characterization and convergence. John Wiley \& Sons.

Jin, Z., Yin, G., \& Zhu, C. (2012). Numerical solutions of optimal risk control and dividend optimization policies under a generalized singular control formulation. Automatica, 48(8), 1489-1501.

Jin, Z., Yang, H., \& Yin, G. (2013). Numerical methods for optimal dividend payment and investment strategies of regime-switching jump diffusion models with capital injections.Automatica, 49(8), 2317-2329.

Kushner, H. J. (1984). Approximation and weak convergence methods for random processes, with applications to stochastic systems theory. MIT press.

Kushner, H. J. (1990). Numerical methods for stochastic control problems in continuous time. SIAM Journal on Control and Optimization, 28(5), 999-1048.

Kushner, H. J., \& Martins, L. F. (1991). Numerical methods for stochastic singular control problems. SIAM journal on control and optimization, 29(6), 1443-1475.

Kushner, H. J., \& Dupuis, P. G. (1992). Numerical methods for stochastic control problems in continuous time. Springer-Verlag.

Kushner, H. (2008). Numerical methods for controlled stochastic delay systems. Springer Science \& Business Media

Liu, M., \& Bai, C. (2015). Optimal harvesting of a stochastic logistic model with time delay. Journal of Nonlinear Science, 25(2), 277-289.

Martins, L. F., \& Kushner, H. J. (1990). Routing and singular control for queueing networks in heavy traffic. SIAM Journal on Control and Optimization, 28(5), 1209-1233.

Radner, R., \& Shepp, L. (1996). Risk vs. profit potential: A model for corporate strategy. Journal of economic dynamics and Control, 20(8), 1373-1393.

Slatkin, M. (1978). The dynamics of a population in a Markovian switching. Ecology, 59, 249-256.

Song, Q., \& Zhu, C. (2012). On Singular Control Problems with State Constraints and RegimeSwitching: A Viscosity Solution Approach, preprint. http://arxiv.org/abs/1208.5757.

Sotomayor, L. R., \& Cadenillas, A. (2011). Classical and singular stochastic control for the optimal dividend policy when there is regime switching. Insurance: Mathematics and Economics, 48(3), 344-354.

Song, Q., Stockbridge, R. H., \& Zhu, C. (2011). On optimal harvesting problems in random environments. SIAM J. Control Optim. 49(2), 859-889.

Tran, K., \& Yin, G. (2014). Stochastic competitive LotkaVolterra ecosystems under partial observation: Feedback controls for permanence and extinction. J. Franklin Inst. 351(8), 4039-4064.

Tran, K., \& Yin, G. (2015). Optimal harvesting strategies for stochastic competitive Lotka-Volterra ecosystems. Automatica, 55, 236-246.

Choulli, T., Taksar, M., \& Zhou, X. Y. (2003). A diffusion model for optimal dividend distribution for a company with constraints on risk control. SIAM Journal on Control and Optimization, 41(6), 1946-1979.

Yang, Z., Yin, G., \& Zhang, Q. (2014). Mean-variance type controls involving a hidden Markov chain: models and numerical approximation. IMA Journal of Mathematical Control and Information, doi: 10.1093/imamci/dnu027.

Yin, G. G., \& Zhang, Q. (2006). Discrete-time Markov chains: two-time-scale methods and applications. Springer
Science \& Business Media.
Yin, G., \& Zhu, C. (2010). Hybrid Switching Diffusions: Properties and Applications, Springer.

Wonham, W. M. (1964). Some applications of stochastic differential equations to optimal nonlinear filtering. SIAM Journal on Control and Optimization, 2(3), 347. 